Mon. Not. R. Astron. Soc. 000,1-?? (0000) Printed 6 April $2021 \quad$ (MN $\mathrm{LTT}_{\mathrm{E}} \mathrm{X}$ style file v2.2)

\title{
Photometric observations of NGC 281: Detection of 228 variable stars
}

\author{
Sneh Lata ${ }^{1 \star}$ A. K. Pandey ${ }^{1}$, J. C. Pandey ${ }^{1}$, Neelam Panwar ${ }^{1}$, Paulomi Paul ${ }^{1}$ \\ ${ }^{1}$ Aryabhatta Research Institute of Observational Sciences, Manora Peak, Nainital 263002, Uttarakhand, India
}

Accepted —. Received —

\begin{abstract}
We identify 228 periodic variables in the region of young open cluster NGC 281 using time series photometry carried out from $1 \mathrm{~m}$ class ARIES telescopes, Nainital. The cluster membership of these identified variables is determined on the basis colourmagnitude, two colour diagrams and kinematic data. Eighty one variable stars are found to be members of the cluster NGC 281. Of 81 variables, 30 and 51 are probable main-sequence and pre-main-sequence members, respectively. Present study classifies main-sequence variable stars into different variability types according to their periods of variability, shape of light curves and location in the Hertzsprung-Russell diagram. These identified main-sequence variables could be $\beta$ Cep, $\delta$ Scuti, slowly pulsating $\mathrm{B}$ type and new class variables. Among 51 pre-main-sequence variables, majority of them are weak line $\mathrm{T}$ Tauri stars. The remaining 147 variables could belong to the field population. The variability characteristics of the field population indicate that these variables could be RR Lyrae, $\delta$ Scuti and binaries type variables.
\end{abstract}

Key words: Open cluster: NGC 281 - colour-magnitude diagram: Variables: premain-sequence stars; $\mathrm{T}$ Tauri stars

\section{INTRODUCTION}

This work presents time series observations of young open cluster NGC 281 as a part of our ongoing project entitled "Search for pre-main-sequence (PMS) variability in Young Open Clusters (YOCs)". YOCs contain significant number of PMS stars with circumstellar disk and are unique laboratories to study the evolution of disks of PMS stars. In general, PMS stars are young low-mass sources which remain deeply embedded the parent molecular cloud. PMS stars show variability associated to a number of different physical processes. Most of the physical processes such as dark spots and flaring, etc are associated with magnetic and rotational activities (Herbst et al. 1994). As a result, PMS variability can be expressed over a wide range of timescales, from intra day to months. These PMS stars accrete matter from their surrounded accretion disk and show prominent features such large IR excess and $\mathrm{H} \alpha$ emission line. In addition to PMS stars, YOCs also contain several O, B and Be type stars which are also found to be pulsating or rotating variables with timescales that range from minutes to years. Among these variables, there are about 20\% B-type stars which show Be phenomenon i.e. B star with emission line.

* E-mail: sneh@aries.res.in
Be type variables are very rapidly rotating main-sequence (MS) stars surrounded by a circumstellar envelope of gas. These stars show variability which is related to the presence of a circumstellar disk of variable size and structure (Arcos et al. 2018).

NGC 281 is a basically HII region known as Sh2-184 and located at a distance of $2.8 \mathrm{kpc}$ (Elmegreen \& Lada 1978; Sato et al. 2008; Sharma et al. 2012). The MS of this cluster is well defined and contains significant number of $\mathrm{O}$ and B spectral type stars. The reddening $E(B-V)$ towards the cluster is found to be low $\sim 0.32$ mag. Previous studies suggest that star formation is still continued in this cluster, hence the cluster provides an excellent laboratory to search PMS for variable stars. A detailed multiwavelength study of the region containing NGC 281 was presented by Sharma et al. (2012). They found that the majority of the identified PMS stars have age $\sim 1-2 \mathrm{Myr}$ and their masses range from 0.5 to $3.5 M_{\odot}$. Recently, Ivers et al. (2014) examined mid and far infrared images of NGC 281 at 24, 70, 100, $160 \mu \mathrm{m}$ wavelength taken from the Herschel and Spitzer Space Observatories to understand the mechanisms of star formation. They found two different populations of protostars in the region of NGC 281. In the WEST of NGC 281, protostars are found to be within a large molecular cloud obscured by dust while other population of protostars is associated with 


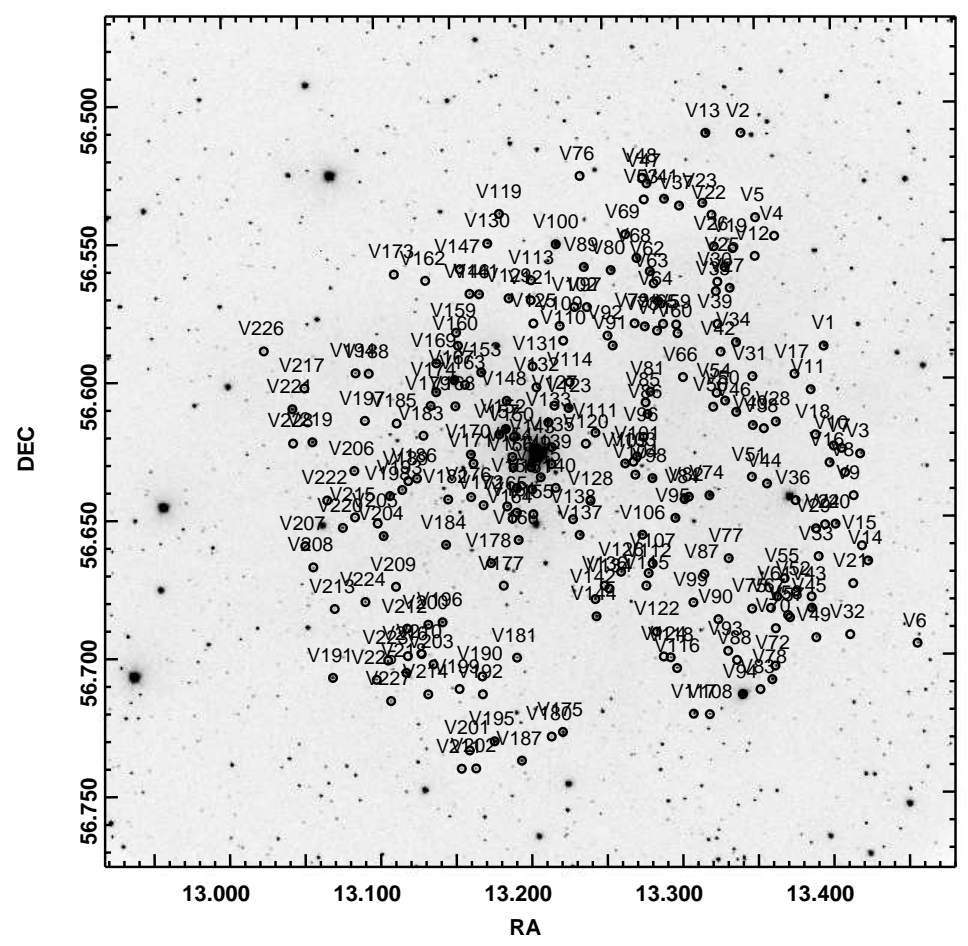

Figure 1. The observed image in $V$ band which contains open cluster NGC 281. The stars detected as variables are encircled.

Table 1. Log of the observations. N and Exp. represent number of frames obtained and exposure time, respectively.

\begin{tabular}{lllll}
\hline S. No. & $\begin{array}{l}\text { Date of } \\
\text { observations }\end{array}$ & Object & $\begin{array}{l}V \\
(\mathrm{~N} \times \mathrm{Exp} .)\end{array}$ & $\begin{array}{l}I \\
(\mathrm{~N} \times \mathrm{Exp} .)\end{array}$ \\
\hline 1 & 28 Oct 2010 & NGC 281 & $4 \times 50 \mathrm{~s}$ & $4 \times 30 \mathrm{~s}$ \\
2 & 30 Oct 2010 & NGC 281 & $4 \times 10 \mathrm{~s}, 60 \mathrm{~s}, 200 \mathrm{~s}, 300 \mathrm{~s}$ & $10 \mathrm{~s}, 40 \mathrm{~s}, 150 \mathrm{~s}$ \\
2 & 30 Oct 2010 & SA 98 & $2 \times 50 \mathrm{~s}, 15 \mathrm{~s}$ & $2 \times 30 \mathrm{~s}, 10 \mathrm{~s}, 50 \mathrm{~s}$ \\
3 & 28 Nov 2011 & NGC 281 & $3 \times 50 \mathrm{~s}$ & - \\
4 & O1 Dec 2011 & NGC 281 & $80 \times 50 \mathrm{~s}$ & - \\
5 & 02 Dec 2011 & NGC 281 & $84 \times 50 \mathrm{~s}$ & - \\
6 & 06 Dec 2012 & NGC 281 & $80 \times 50 \mathrm{~s}$ & - \\
7 & 08 Dec 2012 & NGC 281 & $51 \times 50 \mathrm{~s}$ & - \\
8 & 24 Oct 2014 & NGC 281 & $164 \times 40 \mathrm{~s}$ & - \\
9 & 25 Oct 2014 & NGC 281 & $147 \times 40 \mathrm{~s}$ & - \\
10 & 24 Nov 2014 & NGC 281 & $112 \times 50 \mathrm{~s}$ & - \\
11 & 23 Dec 2014 & NGC 281 & $105 \times 50 \mathrm{~s}$ & - \\
12 & 27 Oct 2017 & NGC 281 & $70 \times 40 \mathrm{~s}$ & \\
13 & 10 Nov 2017 & NGC 281 & $139 \times 40 \mathrm{~s}$ & \\
\hline
\end{tabular}

filamentary pillars and triggered star formation in the East of NGC 281.

We present time series data of stars in the region of the cluster NGC 281. The observations of the field containing NGC 281 were initiated in October 2010 and continued till November 2017. Section 2 describes observations, reduction procedure, comparison of different photometries, variable identification and period determination. Section 3 deals with membership of stars whereas the nature of variable stars identified in the present work has been discussed in section 4 . Finally in section 5, we summarize our results.

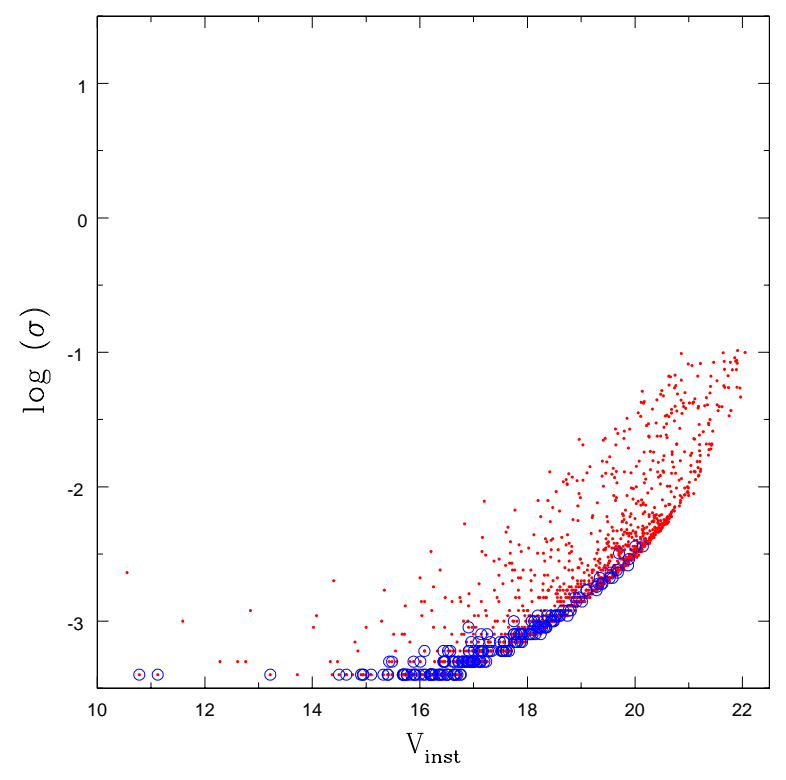

Figure 2. Photometric errors as a function of magnitude. Open circles represent the variables stars identified in the present work.

\section{OBSERVATIONS AND DATA REDUCTION}

Time series $V$ and $I$ band observations of NGC 281 were obtained using a $2048 \times 2048$ pixel CCD camera which is mounted at the f/13 Cassegrain focus of the 1.04-m ARIES Telescope, Nainital, India. The CCD with a plate scale of 


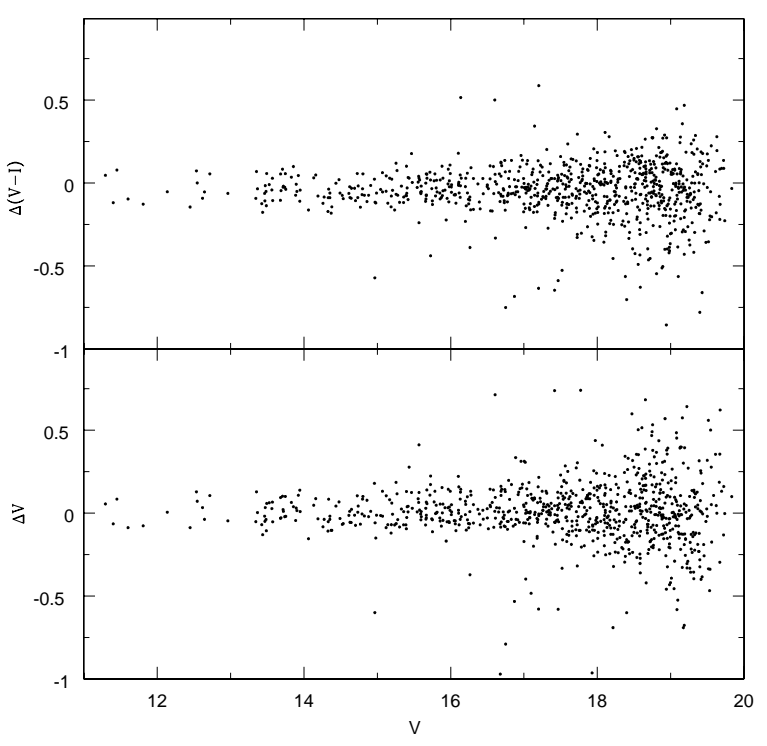

Figure 3. Comparison of the present and previous (Sharma et al. 2012). The $\Delta$ represents the difference (present-previous photometry) between the two photometries.

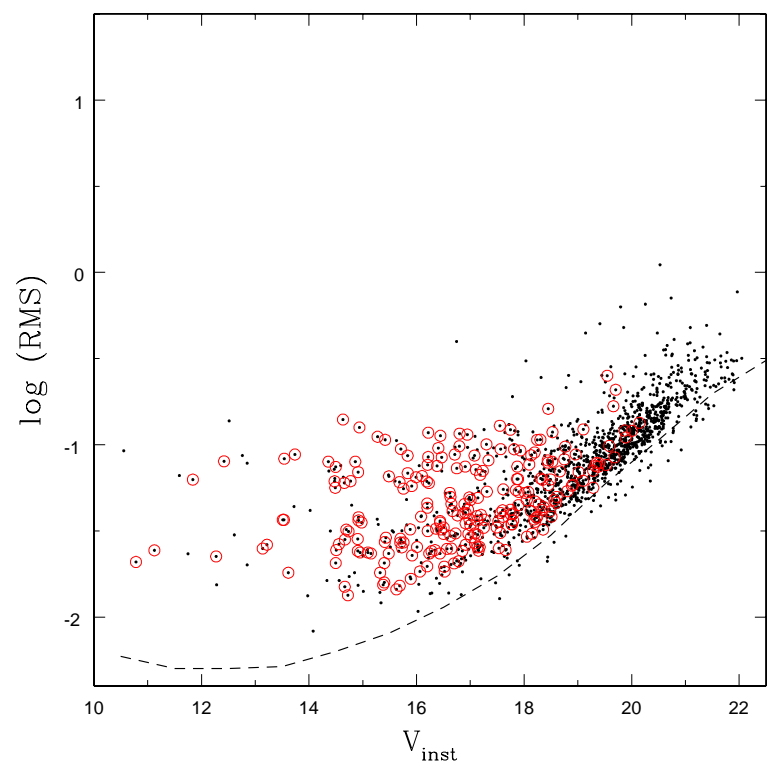

Figure 4. Magnitude as a function of standard deviation of each star detected in the present photometry in $V$ band. Open circles represent variable stars identified in the work. The dashed line shows median magnitude scatter expected from the individual standard errors in different magnitude bins.

$0.37 \mathrm{arcsec} / \mathrm{pixel}$ covers a field of about $13 \times 13 \mathrm{arcmin}^{2}$ on the sky. The observations were carried out in a binning mode of $2 \times 2$ pixels in order to improve the signal-to-noise ratio. During the observations the seeing was around 2 arcsec. To standardize the observations, the SA 98 field of Landolt (1992) was also observed. A number of bias and twilight flat frames were also taken during the observations.

The $V$ band optical observations of NGC 281 were also performed on 27 October 2017 and 10 November 2017 from 1.30-m ARIES Devasthal optical telescope, Nainital, India. This telescope is equipped with $2048 \times 2048$ pixel $^{2}$ CCD camera with a plate scale 0.54 arcsec per pixel, which gives field of view about 18 arcmin $\times 18$ arcmin.

A total of 1046 and 10 observations of the cluster region were secured in $V$ and $I$ bands on 13 and 3 nights, respectively. The log of observations is given in Table 1 . Fig. 1 shows the observed image in $V$ band from $1.30-\mathrm{m}$ ARIES Devasthal optical telescope. The observed images were preprocessed using tasks zerocombine, flatcombine and CCDPROC available in the IRAF1 software package. The DAOPHOT package (Stetson 1987) was used to estimate the instrumental magnitudes. To construct the point spread function (PSF) of images isolated stars across the observed field were selected. The PSF photometry of all the sources was obtained using the ALLSTAR task. DAOMATCH (Stetson 1992) routine of DAOPHOT was used to match up observations taken on various nights with different telescopes. It finds the translation, rotation and scaling solutions between different photometry files, whereas DAOMASTER (Stetson 1992) takes .mch file given by DAOMATCH to match the point sources. DAOMASTER corrects all magnitudes for each star to the magnitude scale of the reference frame. The instrumental magnitudes were translated to the standard magnitudes using the observations of Landolt SA98 standard stars (Landolt 1992). We derived the transformation equations which includes the zero points and colour coefficients as

$$
\begin{gathered}
v=V+a_{1}-b_{1} \times(V-I)+0.25 \times X \\
i=I+a_{2}-b_{2} \times(V-I)+0.10 \times X
\end{gathered}
$$

where $v$ and $i$ are the instrumental magnitudes and $X$ is the airmass. The values of $a_{1}, a_{2}, b_{1}$, and $b_{2}$ are found to be $5.4148645 \pm 0.0037297,5.6550550 \pm 0.0021983,0.0379665 \pm$ 0.0031046 , and $0.0433376 \pm 0.0017998$ respectively.

The standard photometric error of the mean magnitude for each star, based on photometric error of individual frame, has been taken from the .mag file generated by the DAOMASTER. The estimated photometric error of all the stars as a function mean instrumental magnitude is shown in Fig. 2 .

\subsection{Comparison with Previous Photometry}

The present CCD photometric data have been compared with the CCD observations given by Sharma et al. (2012). We have found 867 common stars between these two photometries. The difference $\Delta$ (present data -literature data) as a function of $V$ magnitude is shown in Fig. 3, which indicates that the present $V$ magnitudes are in agreement with those given by Sharma et al. (2012), whereas a systematic difference of $0.05 \mathrm{mag}$ in $(V-I)$ colour is present between the two photometries.

1 IRAF is distributed by the National Optical Astronomy Observatory, which is operated by the Association of Universities for Research in Astronomy (AURA) under cooperative agreement with the National Science Foundation 


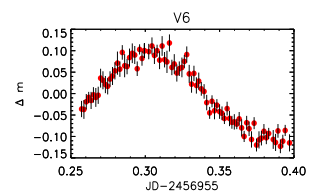

V 15

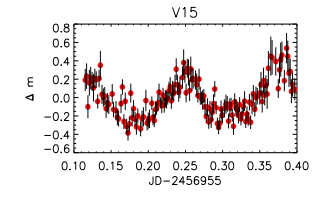

V126

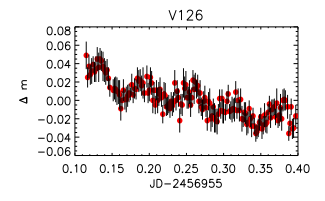

V153

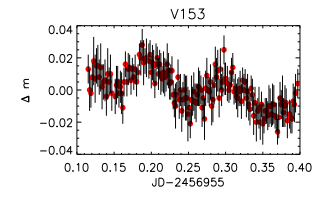

v168

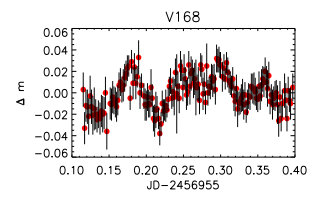

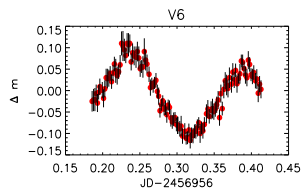

v15

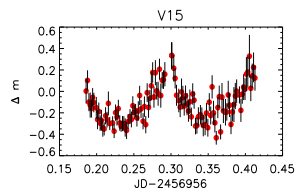

$\mathrm{V} 126$
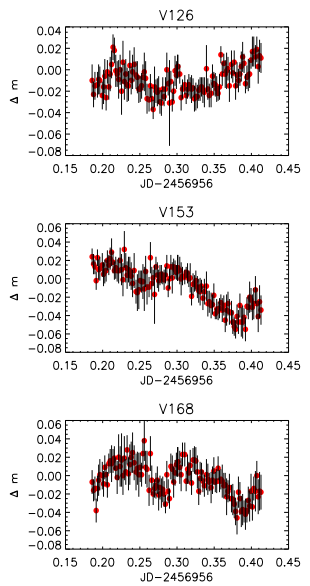
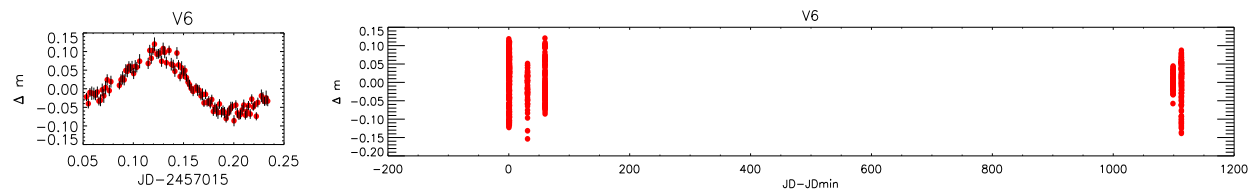

V15

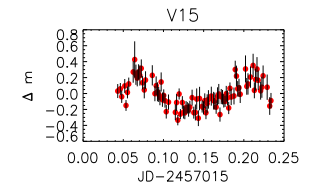

V126

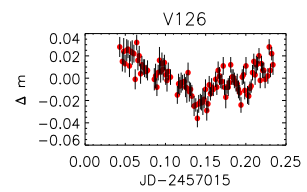

V153

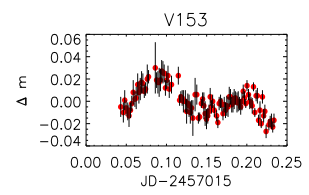

V168

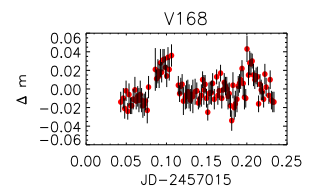

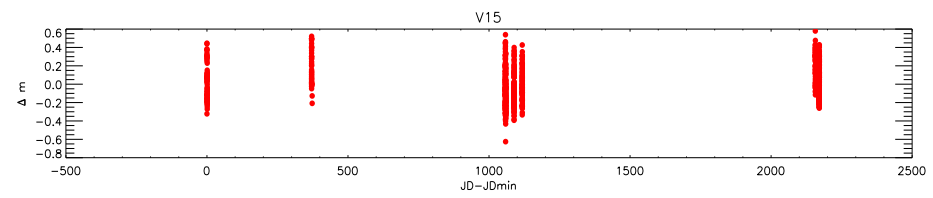

V126
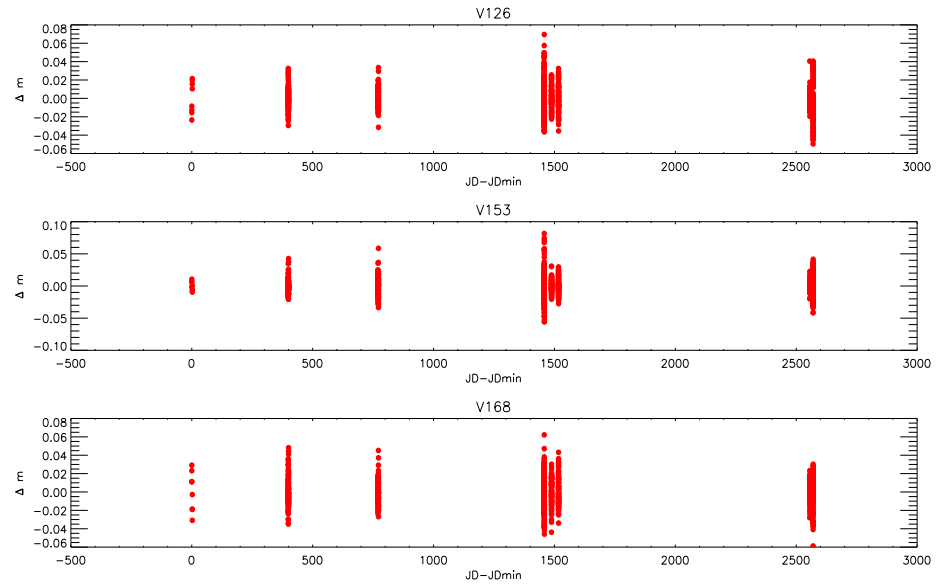

Figure 5. The $V$ band sample light curves of a few variables identified in the present work where $\Delta \mathrm{m}$ represents the differential magnitude. The individual night observations of stars are shown in left panel while right panel represents all the observations of stars. The complete Figure 5 is available in electronic form.

\subsection{Variables identification}

The differential magnitudes $(\Delta \mathrm{m})$ in the sense variable minus comparison star were plotted against Julian date (JD). To obtain differential magnitude of target star $(\mathrm{V})$, we have selected three non variable stars $\mathrm{C} 1, \mathrm{C} 2$ and $\mathrm{C} 3$ as comparison stars in the field of NGC 281. Then, magnitude difference $\mathrm{V}-\mathrm{C} 1, \mathrm{C} 1-\mathrm{C} 2, \mathrm{C} 1-\mathrm{C} 3$ have been determined relative to $\mathrm{C} 1$. The comparison stars $\mathrm{C} 2$ and $\mathrm{C} 3$ have been used to check non variable nature of $\mathrm{C} 1$. The probable variables were identified visually by inspecting the light curves. The visual inspection yielded 228 variable candidates. In order to verify variability of these stars, $\mathrm{rms}$ (root mean square) scatter is computed for each star. The observed rms scatter includes both the intrinsic variability and the mean photometric error. In Fig. 4 we have plotted $r m s$ of each observed star as a function of magnitude, where open circles show the variables identified in the present work. Fig. 4 indicates that generally identified variables have higher $\mathrm{rms}$ value. The lower $r m s$ stars in general are low amplitude variables. There are 4 variables with $r m s>3$ times the mean $r m s$ of the magnitude bin. Ten and 48 variables have $r m s>2$ times the mean rms of the magnitude bin and $r m s>1$ times the mean $\mathrm{rms}$ of the magnitude bin, respectively. The light curves of some of the stars having large rms do not show any variability. These stars are either having some outliers or located on edge of CCD frame. Low amplitude variables have been detected visually by inspecting their light curves. The photometric variability of stars identified as variables has also been checked using the Chi square test (Sesar et al. 2007). All 228 variables have probability $\geqslant 90 \%$ except four stars V129, V136, V139, V141 and V146 for which probability to be a variable is less than $90 \%$. We considered these stars as variable on the basis of visual inspection of their light curves. The sample light curves of a few variables are shown in Fig 5. The identification number, coordinates and optical data for these variable stars are given in Table 2. The CCD pixel coordinates of these identified variables were converted to celestial coordinates (RA and DEC) for J2000 with the help of the CCMAP and CCTRAN tasks in IRAF. The reference astrometric catalog was made from DSS2 $\mathrm{R}$ band image (https://skyview.gsfc.nasa.gov/current/cgi/query.pl) which was used in CCMAP to determine the plate solution for the image. The variable candidates identified in the present work are marked in Fig. 1.

To determine periods of variable stars we applied the Lomb-Scargle (LS) periodogram (Lomb 1976; Scargle 1982). This periodogram is known to work well if data are unevenly sampled. The false alarm probabilities corresponding to the power of maximum frequency from LS for all the identified variables come out to be zero. The peak of the periodogram ranges from 0.076 to 0.92 for variable stars. The range of highest periodogram peak for the present variables is between $\sim 0.021$ to $\sim 0.038, \sim 0.023$ to $\sim 0.040$ and $\sim 0.026$ to $\sim 0.046$ at $10 \%, 5 \%$ and $1 \%$ false alarm probabilities, respectively. The light curves of stars were folded with calculated period. We visually inspected the phased light curves and opted for the period showing the best phased light curve. The most probable periods with amplitude are listed in Table 2. The phased light curves of periodic and semi periodic/irregular variables candidates are shown in Fig. 6 and 


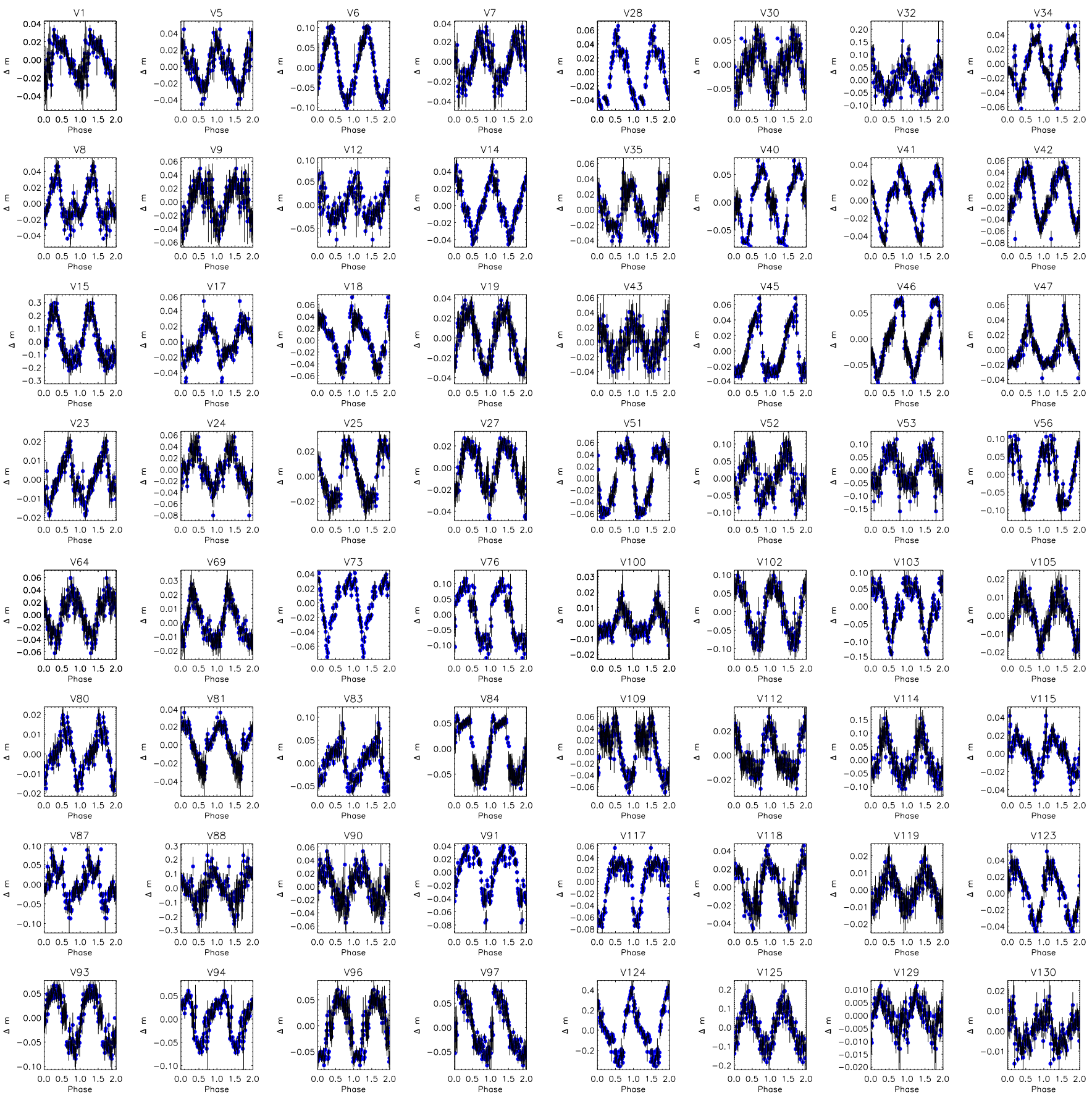

Figure 6. The $V$ band phased light curves of periodic variable stars.

Fig. 7, respectively where averaged differential magnitude in 0.02 phase bin along with $\sigma$ error bar has been plotted. A few of the individual data points which show relatively large photometric errors have not been considered in folding the light curves. During calculation of period for short period variable stars, if there was a vertical shift between the observations for different nights, it was calculated and applied to bring different night observations at same level. In the present study several stars seem to have many periods. These could be semi periodic/irregular variables (light curves shown in Fig. 7). The light curves of these stars were folded with their best periods as folded data with the best period gives smooth phased light curve of the star. These irregular/semiperiodic variables could be binary systems with pulsating components with multiple periods or other complex systems. From now, we have considered them as probable periodic variable stars. The phased light curves will be further discussed in Section 7. 

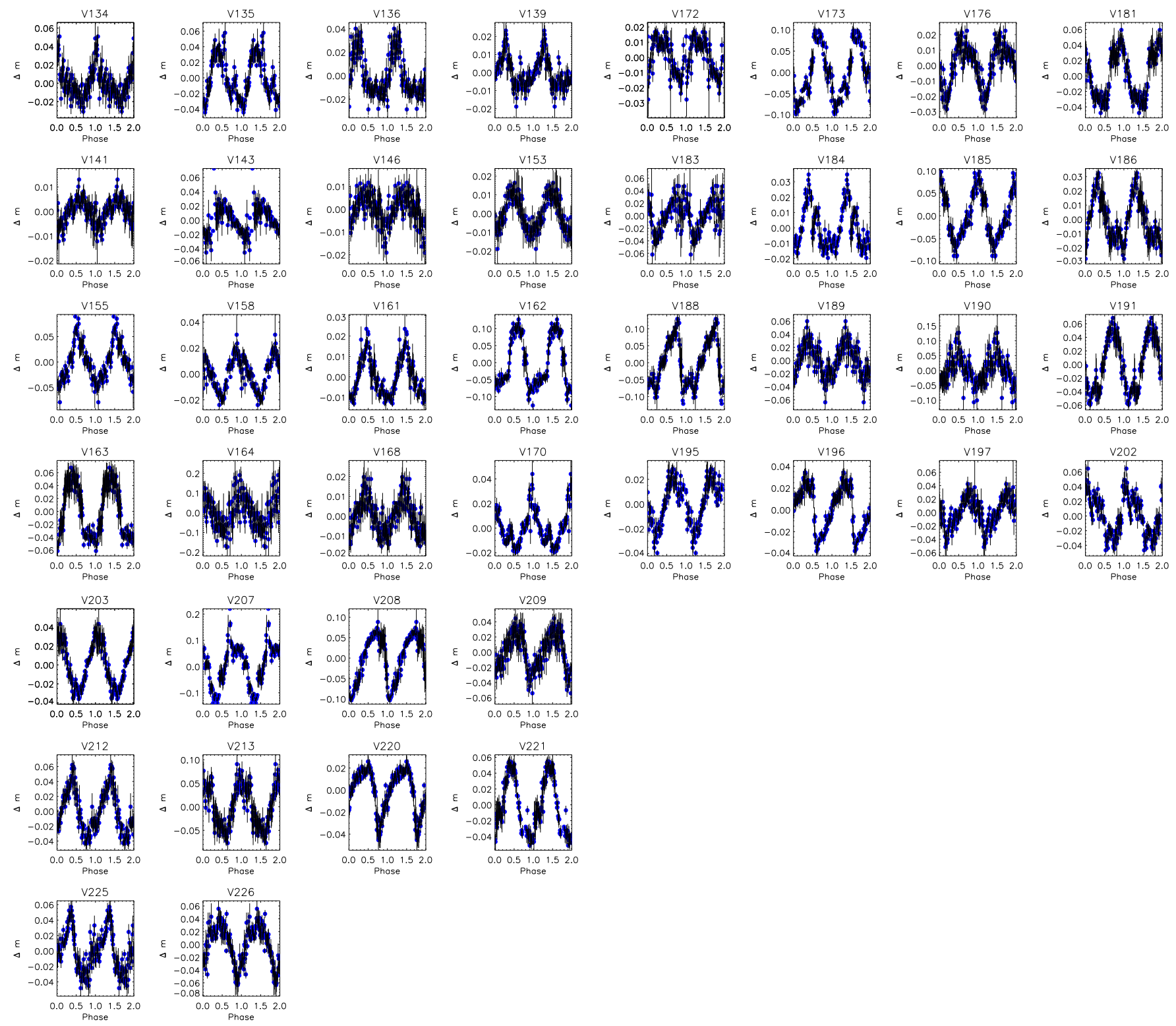

Figure 6. Continued.

\section{CLUSTER MEMBERSHIP OF THE IDENTIFIED VARIABLES}

The characterization of the identified variables needs information whether these are the members of the cluster or not. The $U B V I$ ( $U B V$ data taken Sharma et al. 2012 and present $V I$ data) and $J H K$ photometric observations of the cluster NGC 281 (Cutri et al. 2003) have been used to establish the membership of the identified variables. In addition, the membership of a star is also verified with the help of proper motion data taken from Gaia astrometric mission (Gaia Collaboration et al. 2018)

\subsection{U-B/B-V Two colour diagram (TCD)}

The $U-B / B-V$ TCD is a useful tool to identify MS members of the cluster region. The $U-B$ data for only 128 identified variables are available in Sharma et al. (2012). Fig. 8 plots $U-B / B-V$ TCD for 128 variables where the continuous curves show ZAMS from Girardi et al. (2002), which are shifted along the reddening vector having a slope of $E(U-B) / E(B-V)=0.72$. The distribution of MS variables reveals a variable reddening in the cluster region with a minimum reddening $E(B-V)=0.32 \mathrm{mag}$. The sources lying within the MS band having $E(B-V)=0.32 \mathrm{mag}$ 

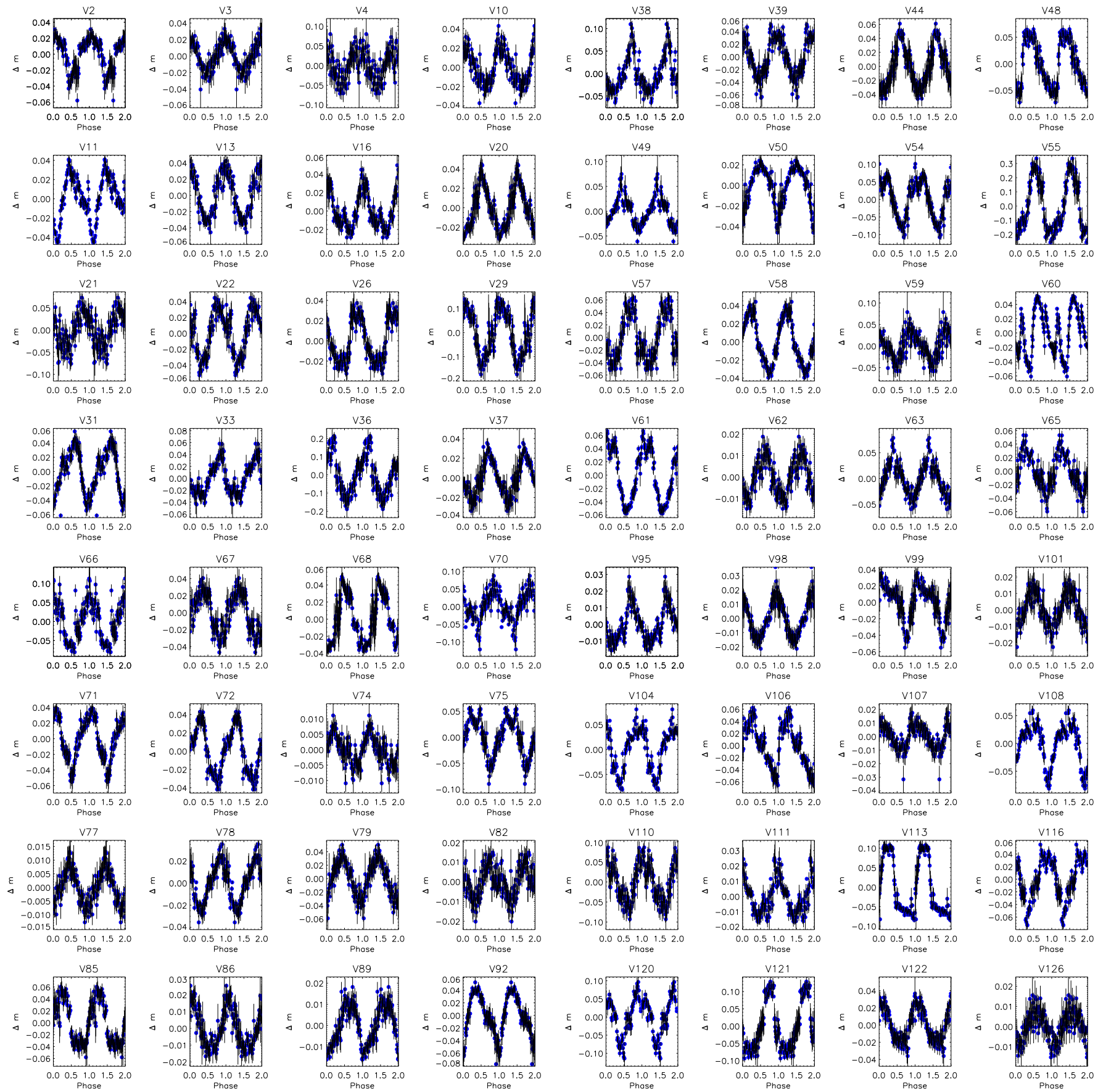

Figure 7. The $V$ band phased light curves of probable periodic variable stars.

to 0.45 mag with $\mathrm{O}$ to A spectral type can be considered as possible MS members of the cluster. The identification as well as estimation of reddening for YSOs is not possible using the $U-B / B-V$ TCD because the $U$ and $B$ band fluxes may be affected by excess due to accretion. Probable MS cluster members are mentioned in Table 2 .

\section{$3.2 \quad \mathrm{~J}-\mathrm{H} / \mathrm{H}-\mathrm{K}$ TCD}

Since young stellar objects (YSOs) generally show $H \alpha$ emission, NIR excess or X-ray emission, therefore $J-$
$H / H-K$ TCD is one of the very useful tools to identify PMS objects. Fig. 9 displays $J-H / H-K$ TCD for all the identified variables. $J H K$ data have been taken from the 2MASS catalogue (Cutri et al. 2003) which was further converted to CIT system using the relations given in the 2MASS website (http://www.astro.caltech.edu/ $\mathrm{jmc} / 2 \mathrm{mass} / \mathrm{v} 3 /$ transformations/). In Fig. 9, the sources lying in ' $\mathrm{F}$ ' region could be either field stars or Class III (WTTSs) and Class II (CTTSs) sources with small NIR excesses. The sources lying in the ' $\mathrm{T}$ ' region can be considered mostly as Class II objects/ CTTSs (for detail, Lata et al. 

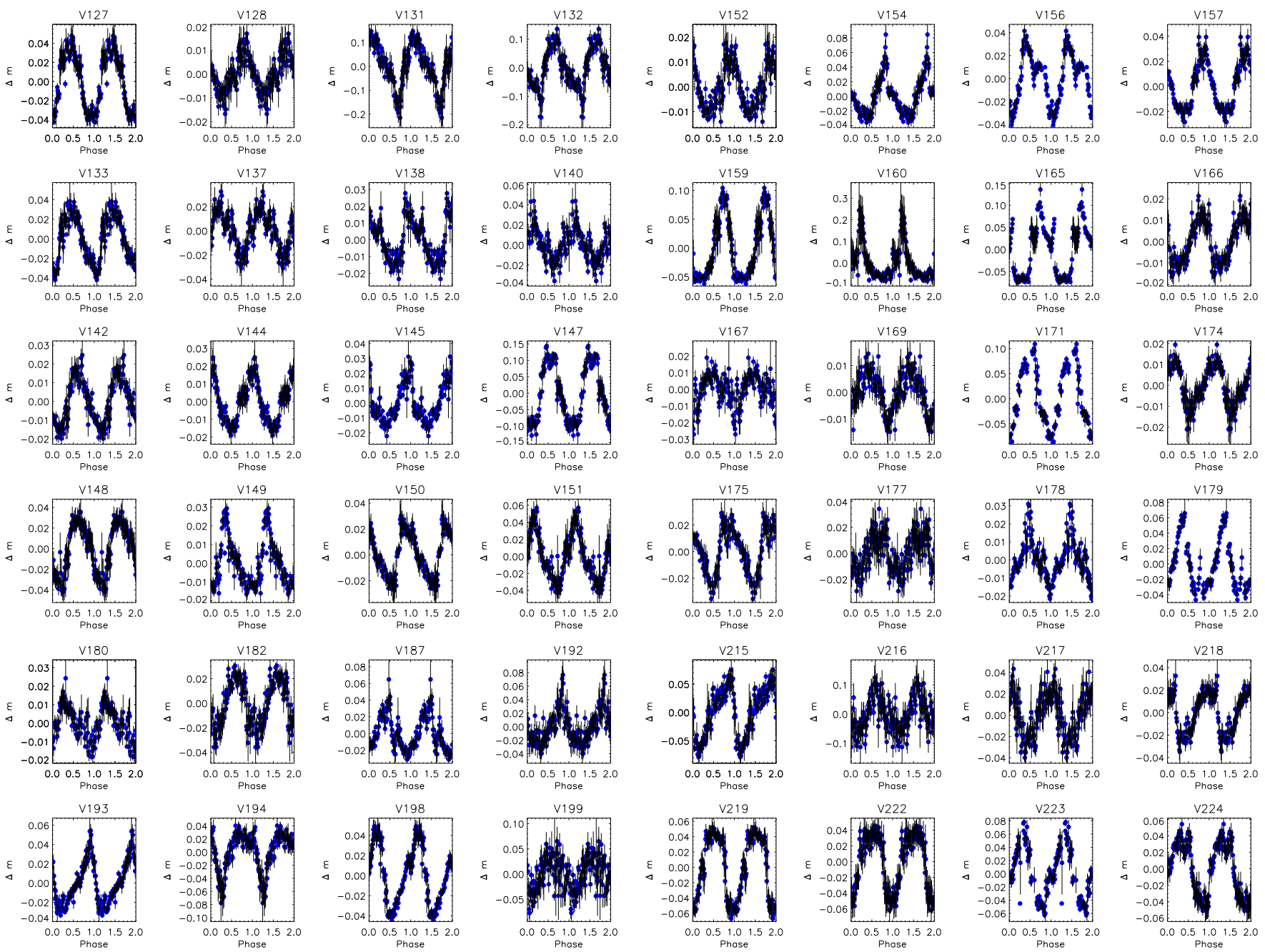
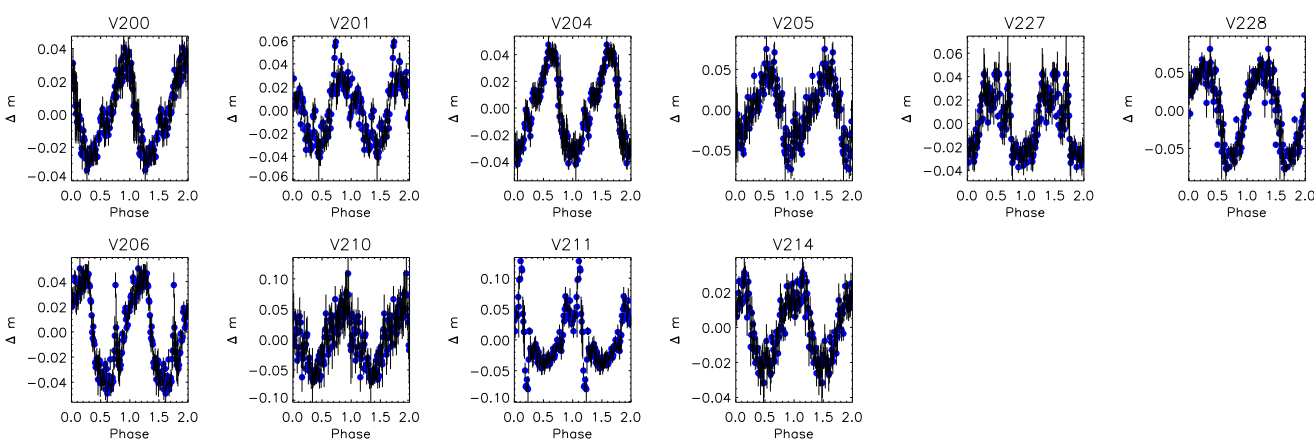

Figure 7. Continued.

2019). A comparison of the TCD of the NGC 281 region with the NIR TCD of nearby reference region (figure 6 of Sharma et al. 2012) indicates that the sources lying in the ' $F$ ' region above the extension of the intrinsic CTTS locus as well as sources having $(J-H) \gtrsim 0.6 \mathrm{mag}$ and lying to the left of the first (left-most) reddening vector could be WTTSs/Class III sources. Sharma et al. (2012) have also identified YSOs on the basis of $H \alpha$ emission, NIR TCD, MIR TCD, and X-ray emission. A comparison of present data with that by Sharma et al. (2012) yields 11 common PMS stars. The MIR observations are very useful, which provide information of YSOs remained embedded in the molecular clouds. Several studies (Getman et al. 2012; Jose et al. 2013) classify YSOs with help of MIR TCDs. In the IRAC colour plane, YSOs occupy distinct regions according to their nature. The figure 7 of Sharma et al. (2012) presents a [5.8]-[8.0] versus [3.6]-[4.5] TCD for the observed sources. This figure shows that class II, III and 0/I sources have different locations in these TCDs. The common YSOs are V76, V102, V103, V114, V133, V152, V156, V164, V171, V188 and V209. In $J-H / H-K$ TCD star V152 lies below the intrinsic locus of T Tauri stars and right of middle reddening vector. Out of these 11 stars two 


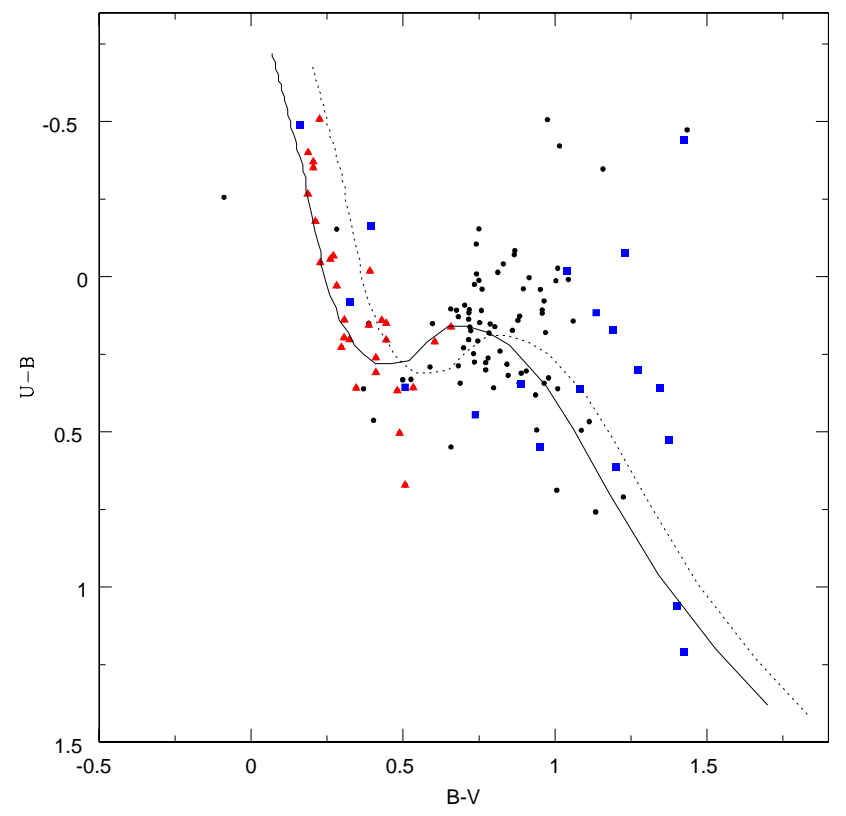

Figure 8. $(U-B) /(B-V)$ TCD for variable stars identified in the present study. All the $U B V$ data are taken from Sharma et al. (2012). The continuous and dotted line represent the ZAMS (Girardi et al. 2002) which are shifted along the reddening vector for reddening $E(B-V)=0.32 \mathrm{mag}$ and $0.45 \mathrm{mag}$. Triangles are identified as MS variables and filled squares show PMS variables.

sources V133 and V156 are found to be lying in the location of Herbig Ae/Be stars in $J-H / H-K$ TCD. Details of the probable PMS cluster members are mentioned in Table 2.

\subsection{V/V-I CMD}

The $V / V-I$ CMD for 225 identified variables is displayed in Fig. 10. The $V-I$ colours for three variables were not available. The zero-age main-sequence (ZAMS) by Girardi et al. (2002) as well as PMS isochrones for various ages and evolutionary tracks for various masses by Siess et al. (2000) have also been plotted. Assuming the reddening $E(V-I)$ towards the cluster as $0.40 \mathrm{mag}$, the comparison of observations with the ZAMS by Girardi et al. (2002) yields a distance modulus of $13.25 \mathrm{mag}$, which corresponds a distance of $2.82 \mathrm{kpc}$, which is in good agreement with the value (2.81 kpc) used by Sharma et al. (2008). The probable MS, PMS variables identified on the basis of CMD are shown with triangle and filled square symbols in Fig. 10, respectively. The variables having membership probabilities $\geqslant 50 \%$ (see next section) are encircled. Based on the above mentioned TCDs and CMD, we have established membership of 81 stars, of which 30 and 51 stars could be probable MS and PMS variables, respectively. Remaining 147 variables may belong to the field region. The classification of variables is given in the last column of Table 2.

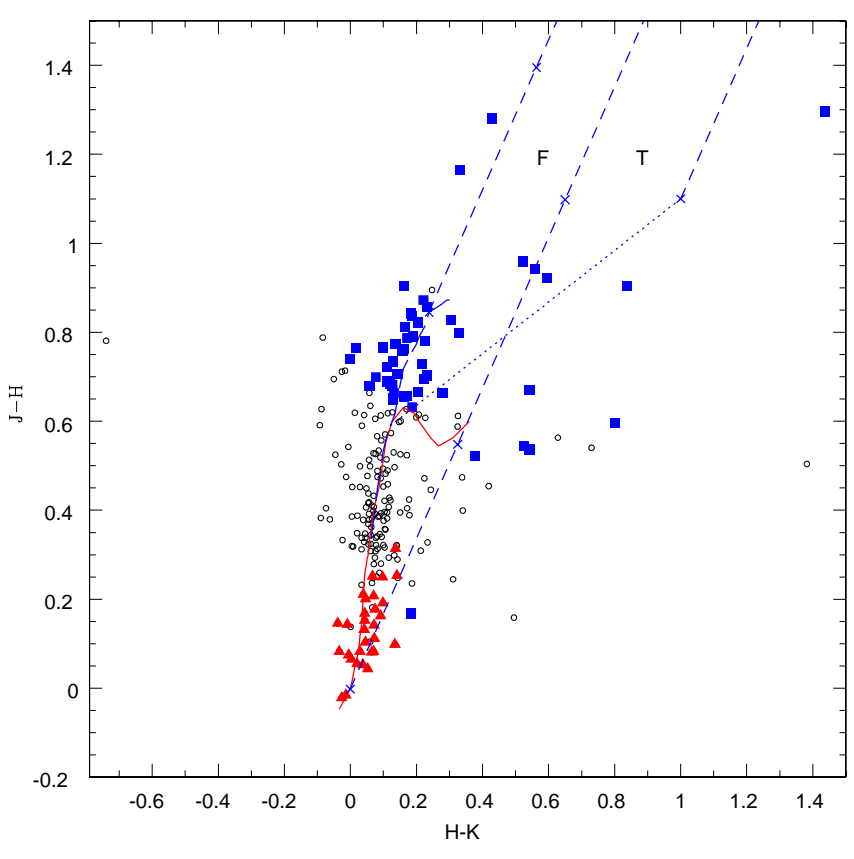

Figure 9. $(J-H) /(H-K)$ TCD for variable stars detected in the field of NGC 281. The $J H K$ data have been taken from the 2MASS catalogue (Cutri et al. 2003). The continuous and long dashed line show sequences for dwarfs and giants (Bessell \& Brett 1988), respectively. The TTSs locus (Meyer et al. 1997) is shown by dotted line. The small dashed lines are reddening vectors (Cohen et al. 1981) and an increment of visual extinction of $A_{V}=5 \mathrm{mag}$ is denoted by crosses on the reddening vectors. Filled squares and triangles represent PMS and MS whereas open circles may be either MS members of the cluster or field stars. The ' $\mathrm{F}$ ' and ' $\mathrm{T}$ ' regions are explained in Section 3.2.

\subsection{Kinematic data}

The proper motion values of stars have been taken from Gaia astrometric mission (Gaia Collaboration et al. 2018). For this we have taken data of stars which lie within radial distance of 16 arcmin from center of the cluster NGC 281. The center of the cluster is assumed at $\mathrm{RA}=00 \mathrm{~h} 52 \mathrm{~m} 49 \mathrm{~s}$ and Dec $=56 \mathrm{~d} 37 \mathrm{~m} 48 \mathrm{~s}$ (Sharma et al. 2012). For the membership probabilities of these stars, we used the online Clusterix membership (http://clusterix.cerit-sc.cz/) estimation tool which uses the Non-Parametric method for the clusterfield separation in 2-dimensional proper motion space. This approach uses the kernel estimation technique using a circular Gaussian kernel function to derive the data distributions as described by Cabrera-Cano \& Alfaro (1990). The fundamental idea underlying this approach is the empirical determination of the cluster and field star distributions without any assumption regarding their shape. The non-parametric method works best for the open clusters where it is somewhat difficult to locate the center of the field region. The proper motion cut-off was set to $15 \mathrm{mas} / \mathrm{yr}$ and radius of the cluster was considered as 8.0 arcmin. For field region we have considered a region lying between 8 and 16 arcmin. Clusterix gave membership probabilities of 3748 stars. The cross matched between present data and data (proper motions and membership probabilities of stars) given by Clusterix reveals that 223 identified variables have membership 


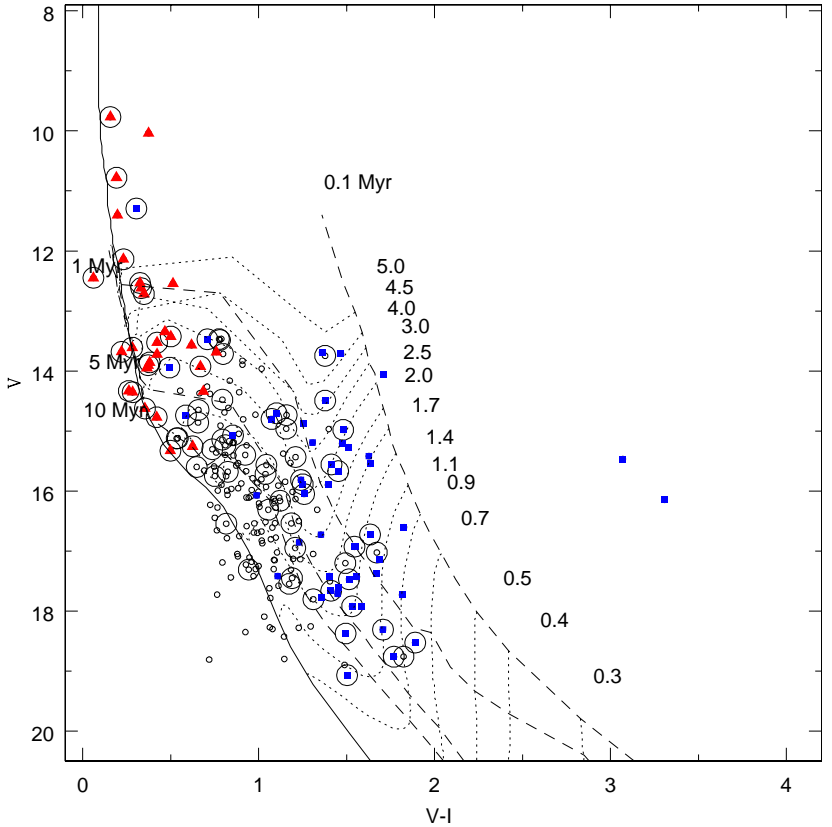

Figure 10. $V /(V-I) \mathrm{CMD}$ for variable stars in the region of the cluster NGC 281. The filled squares represent probable PMS variable stars, whereas triangles show MS stars. The variable stars belong to the field population is shown by open circles. The encircled points here refer to those stars which are having membership probability $\geqslant 50 \%$. The continuous curve shows ZAMS by Girardi et al. (2002) while dashed lines represent PMS isochrones for 0.1, 1, 5, 10 Myrs (Siess et al. 2000). The PMS evolutionary tracks for different masses taken from Siess et al. (2000) are shown by dotted curves.

probability. The membership probability of variable stars along with proper motion values is given in Table 2. Fig. 11 shows the vector point diagram (VPD) of the proper motions for stars of the NGC 281. The highest concentrated area in the VPD gives 44 of 81 members identified from TCDs and CMD, and these 44 stars could be the most probable members of cluster. The 44 members distributed in the highest concentrated area in Fig. 11 have membership probabilities $\geqslant 50 \%$. Out of 81 members, 37 stars with membership probabilities $<50 \%$ could be considered as the least probable members of the cluster because of their locations in $V / V-I$ CMD and $J-H / H-K$ TCD. There are 35 field stars with membership probabilities $\geqslant 50 \%$. Though the membership probabilities of all these 35 stars are $\geqslant 50 \%$ and 33 of which are located where PMS stars are found to be lying in $V / V-I \mathrm{CMD}$, they could not be considered as members of the cluster because of their locations in $J-H / H-K$ TCD.

\section{NATURE OF VARIABLE STARS}

Of 228 periodic variables detected in the present work, 81 (30 MS and 51 PMS) stars are found to be associated with the cluster. The MS variable stars are classified according to their periods of variability, shape of light curves, as well as their locations in the Hertzsprung - Russell (HR) diagram. The $H-R$ diagram for MS variables is shown in Fig. 12. The

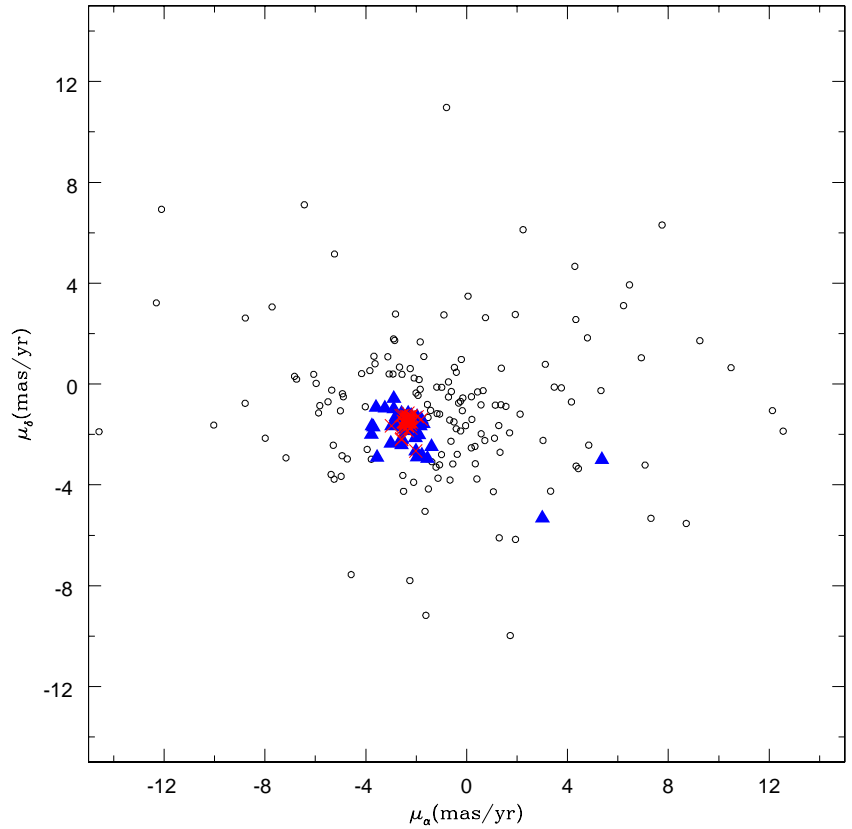

Figure 11. The VPD of the proper motions for stars in the NGC 281 region. Open circles represent proper motions of 223 stars (cf. section 3.4) while triangles represent variables with membership probabilities $\geqslant 50 \%$. The crosses show present cluster members (both MS and PMS) with membership probabilities $\geqslant 50 \%$.

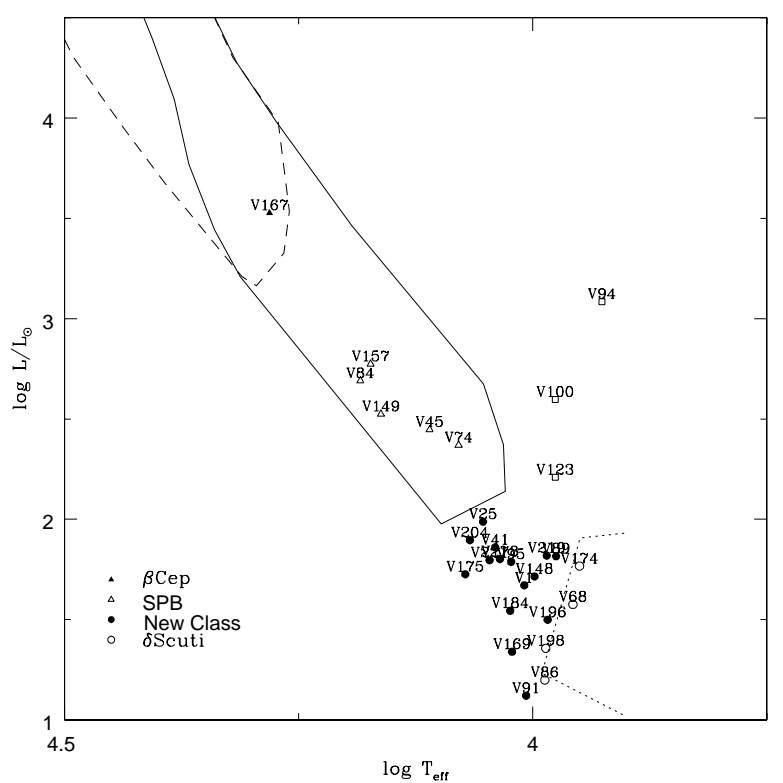

Figure 12. $\log \left(L / L_{\odot}\right) / \log T_{\text {eff }}$ diagram for the probable MS variable stars identified in the present study. The continuous curve represents the instability strip of SPB stars where as dotted curves shows the instability region of $\delta$ Scuti stars. The dashed curve shows the location of $\beta$ Cep stars (cf. Balona et al. 2011). 
Table 2. The VI data, amplitude and period of variables in the open cluster NGC 281. The Proper motion data were taken from Gaia (Gaia Collaboration et al. 2018). The column Prob. refers to the membership probabilities of variable stars calculated using proper motion data.

\begin{tabular}{|c|c|c|c|c|}
\hline ID & RA(2000) & $\operatorname{Dec}(2000)$ & $\underset{(\operatorname{mag})}{V}$ & $\begin{array}{c}I \\
\text { (mag) }\end{array}$ \\
\hline V1 & 13.394370 & 56.588220 & $13.961 \pm 0.018$ & $12.944 \pm 0.021$ \\
\hline $\mathrm{V} 2$ & 13.340740 & 56.511100 & $15.530 \pm 0.024$ & $14.349 \pm 0.013$ \\
\hline V3 & 13.418240 & 56.627450 & $15.534 \pm 0.020$ & $14.650 \pm 0.021$ \\
\hline $\mathrm{V} 4$ & 13.362610 & 56.548570 & $18.254 \pm 0.051$ & $16.958 \pm 0.018$ \\
\hline V5 & 13.349920 & 56.541820 & $16.535 \pm 0.026$ & $15.442 \pm 0.008$ \\
\hline V6 & 13.455430 & 56.695880 & $15.845 \pm 0.023$ & $14.694 \pm 0.009$ \\
\hline $\begin{array}{l}\text { V7 } \\
\text { V8 }\end{array}$ & $\begin{array}{l}13.405920 \\
13.408430\end{array}$ & $\begin{array}{l}56.6253330 \\
56.634080\end{array}$ & $\begin{array}{l}16.091 \pm 0.018 \\
16.162 \pm 0.018\end{array}$ & $\begin{array}{l}15.137 \pm 0.011 \\
15.040 \pm 0.011\end{array}$ \\
\hline $\begin{array}{l}\text { V8 } \\
\text { V9 }\end{array}$ & $\begin{array}{l}13.408430 \\
13.413880\end{array}$ & $\begin{array}{l}56.634080 \\
56.642560\end{array}$ & $\begin{array}{l}16.162 \pm 0.018 \\
17.391 \pm 0.037\end{array}$ & $15.040 \pm 0.011$ \\
\hline V10 & 13.400810 & 56.624510 & $16.165 \pm 0.020$ & $15.073 \pm 0.014$ \\
\hline V11 & 13.385840 & 56.604010 & $15.376 \pm 0.021$ & $14.236 \pm 0.017$ \\
\hline V12 & 13.349280 & 56.555970 & $17.728 \pm 0.029$ & $15.912 \pm 0.398$ \\
\hline $\begin{array}{l}\text { V13 } \\
\text { V14 }\end{array}$ & 13.317420 & $\begin{array}{l}56.511180 \\
56666444\end{array}$ & $12.224 \pm 0.019$ & \\
\hline $\begin{array}{l}\text { V14 } \\
\text { V15 }\end{array}$ & $\begin{array}{l}13.423000 \\
13.419056\end{array}$ & $\begin{array}{l}56.666444 \\
56.661000\end{array}$ & $\begin{array}{l}14.371 \pm 0.008 \\
18.807+0.242\end{array}$ & $\begin{array}{l}13.707 \pm 0.010 \\
18.088+0.065\end{array}$ \\
\hline V16 & 13.397806 & 56.630806 & $\begin{array}{l}18.807 \pm 0.242 \\
15.236 \pm 0.008\end{array}$ & $14.425 \pm 0.008$ \\
\hline V17 & 13.375028 & 56.598694 & $15.991 \pm 0.010$ & $15.223 \pm 0.012$ \\
\hline V18 & 13.388667 & 56.620694 & $15.668 \pm 0.011$ & $14.215 \pm 0.009$ \\
\hline V19 & 13.334667 & 56.553167 & $16.538 \pm 0.041$ & $15.352 \pm 0.010$ \\
\hline $\begin{array}{l}\text { V20 } \\
\text { V21 }\end{array}$ & $\begin{array}{l}13.401583 \\
13.413111\end{array}$ & $\begin{array}{l}56.653056 \\
56.674639\end{array}$ & $\begin{array}{l}14.328 \pm 0.006 \\
17.765+0.032\end{array}$ & $\begin{array}{l}13.774 \pm 0.008 \\
16.409+0.014\end{array}$ \\
\hline $\begin{array}{l}\mathrm{V} 21 \\
\mathrm{~V} 22\end{array}$ & $\begin{array}{l}13.413111 \\
13.321028\end{array}$ & $\begin{array}{l}56.674639 \\
56.541028\end{array}$ & $\begin{array}{l}17.765 \pm 0.032 \\
16.825 \pm 0.025\end{array}$ & $\begin{array}{l}16.409 \pm 0.014 \\
15.807 \pm 0.013\end{array}$ \\
\hline V23 & 13.315000 & 56.536750 & $14.161 \pm 0.006$ & $13.325 \pm 0.009$ \\
\hline V24 & 13.394694 & 56.653278 & $17.153 \pm 0.021$ & $16.062 \pm 0.011$ \\
\hline V25 & 13.329500 & 56.559972 & $13.526 \pm 0.005$ & $13.103 \pm 0.006$ \\
\hline V26 & 13.322417 & 56.552472 & $15.908 \pm 0.013$ & $14.796 \pm 0.009$ \\
\hline $\begin{array}{l}\text { V27 } \\
\text { V28 }\end{array}$ & $\begin{array}{l}13.332667 \\
13.362556\end{array}$ & $\begin{array}{l}56.567500 \\
56.616000\end{array}$ & $\begin{array}{l}14.648 \pm 0.007 \\
15.407+0.01\end{array}$ & $\begin{array}{l}13.990 \pm 0.007 \\
14.550+0.012\end{array}$ \\
\hline $\begin{array}{l}\text { V28 } \\
\text { V29 }\end{array}$ & $\begin{array}{l}13.362556 \\
13.388639\end{array}$ & $\begin{array}{l}56.616000 \\
56.654694\end{array}$ & $\begin{array}{l}15.407 \pm 0.010 \\
17.928 \pm 0.039\end{array}$ & $\begin{array}{l}14.550 \pm 0.012 \\
16.342 \pm 0.031\end{array}$ \\
\hline V30 & 13.324583 & 56.565278 & $17.918 \pm 0.066$ & $16.386 \pm 0.013$ \\
\hline V31 & 13.347389 & 56.599528 & $15.858 \pm 0.023$ & $14.865 \pm 0.008$ \\
\hline V32 & 13.410944 & 56.693083 & $18.270 \pm 0.045$ & $17.206 \pm 0.025$ \\
\hline V33 & $\begin{array}{l}13.390361 \\
136667\end{array}$ & $\begin{array}{l}56.66475 \\
56587139\end{array}$ & $16.673 \pm 0.017$ & $15.657 \pm 0.012$ \\
\hline $\begin{array}{l}\text { V344 } \\
\text { V35 }\end{array}$ & $\begin{array}{l}13.336667 \\
13.323556\end{array}$ & $\begin{array}{r}56.587139 \\
56.568694\end{array}$ & $\begin{array}{l}13.894 \pm 0.009 \\
16.602+0.017\end{array}$ & $\begin{array}{l}12.986 \pm 0.006 \\
14.775+0.011\end{array}$ \\
\hline $\begin{array}{l}\text { V35 } \\
\text { V36 }\end{array}$ & $\begin{array}{l}13.323556 \\
13.375361\end{array}$ & $\begin{array}{l}56.568694 \\
56.644583\end{array}$ & $\begin{array}{l}16.602 \pm 0.017 \\
17.438 \pm 0.062\end{array}$ & $\begin{array}{l}14.775 \pm 0.011 \\
16.596 \pm 0.032\end{array}$ \\
\hline V37 & 13.299611 & 56.537639 & $14.727 \pm 0.007$ & $13.745 \pm 0.008$ \\
\hline V38 & 13.354639 & 56.618389 & $17.470 \pm 0.033$ & $15.956 \pm 0.012$ \\
\hline V39 & 13.324583 & 56.580528 & $17.144 \pm 0.034$ & $15.457 \pm 0.008$ \\
\hline $\begin{array}{l}\text { V40 } \\
\text { Y } 41\end{array}$ & $\begin{array}{l}13.347361 \\
13.289889\end{array}$ & $\begin{array}{l}56.617222 \\
56535167\end{array}$ & $\begin{array}{l}15.168 \pm 0.017 \\
13682+0.008\end{array}$ & $14.335 \pm 0.007$ \\
\hline $\begin{array}{l}\text { V41 } \\
\text { V42 }\end{array}$ & $\begin{array}{l}13.289889 \\
13.326899\end{array}$ & $\begin{array}{l}56.535167 \\
56.590611\end{array}$ & $\begin{array}{l}13.682 \pm 0.008 \\
16.11 \pm 0.029\end{array}$ & $\begin{array}{l}12.922 \pm 0.006 \\
15.167 \pm 0.013\end{array}$ \\
\hline $\begin{array}{l}\text { V42 } \\
\text { V43 }\end{array}$ & $\begin{array}{l}13.326389 \\
13.385611\end{array}$ & 56.679278 & $\begin{array}{l}16.11 \pm 0.029 \\
17.504 \pm 0.051\end{array}$ & $\begin{array}{l}15.099 \pm 0.013 \\
16.099 \pm 0.013\end{array}$ \\
\hline V44 & 13.356528 & 56.638389 & $14.966 \pm 0.007$ & $13.567 \pm 0.006$ \\
\hline V45 & 13.385694 & 56.683417 & $12.448 \pm 0.008$ & $12.387 \pm 0.012$ \\
\hline V46 & 13.336500 & 56.612444 & $13.829 \pm 0.013$ & $12.921 \pm 0.009$ \\
\hline $\begin{array}{l}\text { V47 } \\
\text { Y } 48\end{array}$ & $\begin{array}{l}13.278389 \\
13.275667\end{array}$ & $\begin{array}{l}56.529556 \\
56527500\end{array}$ & $\begin{array}{l}13.746 \pm 0.012 \\
17048+0.044\end{array}$ & $\begin{array}{l}12.368 \pm 0.009 \\
15.738+0.019\end{array}$ \\
\hline $\begin{array}{l}\text { V48 } \\
\text { V49 }\end{array}$ & $\begin{array}{l}13.275667 \\
13.388472\end{array}$ & $\begin{array}{l}56.527500 \\
56.694111\end{array}$ & $\begin{array}{l}17.048 \pm 0.044 \\
16.072+0.011\end{array}$ & $\begin{array}{l}15.738 \pm 0.011 \\
15.083 \pm 0.009\end{array}$ \\
\hline V50 & 13.329250 & 56.608333 & $14.804 \pm 0.008$ & $\begin{array}{l}15.083 \pm 0.009 \\
13.892 \pm 0.006\end{array}$ \\
\hline V51 & 13.346583 & 56.635944 & $16.205 \pm 0.013$ & $15.203 \pm 0.008$ \\
\hline V52 & 13.375139 & 56.677611 & $18.425 \pm 0.060$ & $17.280 \pm 0.049$ \\
\hline $\begin{array}{l}\text { V53 } \\
\text { V54 }\end{array}$ & 13.276667 & 56.535389 & $18.757 \pm 0.074$ & $16.933 \pm 0.023$ \\
\hline $\begin{array}{l}\text { V54 } \\
\text { V555 }\end{array}$ & $\begin{array}{l}13.323639 \\
13.367833\end{array}$ & $\begin{array}{l}56.605444 \\
566672722\end{array}$ & $\begin{array}{l}17.450 \pm 0.092 \\
18\end{array}$ & $\begin{array}{ll}16.260 \pm 0.011 \\
16.601+0.013\end{array}$ \\
\hline $\begin{array}{l}\text { V55 } \\
\text { V56 }\end{array}$ & $\begin{array}{l}13.367833 \\
13.321417\end{array}$ & $\begin{array}{l}56.672722 \\
56.610500\end{array}$ & $\begin{array}{l}\begin{array}{l}18.308 \pm 0.053 \\
17.201 \pm 0.020\end{array}\end{array}$ & $\begin{array}{l}16.601 \pm 0.013 \\
15.708 \pm 0.013\end{array}$ \\
\hline V57 & 13.371333 & 56.686944 & $17.023 \pm 0.027$ & $15.351 \pm 0.029$ \\
\hline V58 & 13.369972 & 56.685972 & $15.419 \pm 0.009$ & $13.792 \pm 0.009$ \\
\hline V59 & 13.297333 & 56.580694 & $17.802 \pm 0.037$ & $16.491 \pm 0.012$ \\
\hline $\begin{array}{l}\text { V60 } \\
\text { V61 }\end{array}$ & 13.298278 & 56.583806 & $16.130 \pm 0.017$ & $15.044 \pm 0.006$ \\
\hline $\begin{array}{l}\text { V61 } \\
\text { V62 }\end{array}$ & $\begin{array}{l}13.362944 \\
13.280194\end{array}$ & $\begin{array}{l}56.679222 \\
56.561361\end{array}$ & $\begin{array}{l}15.964 \pm 0.014 \\
13.715 \pm 0.014\end{array}$ & $\begin{array}{l}15.119 \pm 0.016 \\
12.917 \pm 0.008\end{array}$ \\
\hline $\begin{array}{l}\mathrm{V} 62 \\
\mathrm{~V} 63\end{array}$ & $\begin{array}{l}13.280194 \\
13.282667\end{array}$ & $\begin{array}{l}56.561361 \\
56.565722\end{array}$ & $\begin{array}{l}13.715 \pm 0.014 \\
17.248 \pm 0.024\end{array}$ & $\begin{array}{l}12.917 \pm 0.008 \\
16.248 \pm 0.016\end{array}$ \\
\hline V64 & 13.285611 & 56.572167 & $15.434 \pm 0.011$ & $14.224 \pm 0.006$ \\
\hline V65 & 13.288722 & 56.580389 & $16.583 \pm 0.024$ & $15.651 \pm 0.009$ \\
\hline V66 & 13.301639 & 56.599722 & $17.546 \pm 0.037$ & $16.372 \pm 0.023$ \\
\hline V67 & 13.358639 & 56.683389 & $16.750 \pm 0.018$ & $15.860 \pm 0.011$ \\
\hline V68 & 13.271528 & 56.556500 & $13.949 \pm 0.007$ & $13.584 \pm 0.006$ \\
\hline V69 & 13.264167 & 56.547889 & $14.910 \pm 0.007$ & $14.127 \pm 0.007$ \\
\hline V70 & 13.361861 & 56.690722 & $17.783 \pm 0.04$ & $16.716 \pm 0.017$ \\
\hline V71 & 13.284639 & 56.582861 & $15.994 \pm 0.010$ & $15.173 \pm 0.012$ \\
\hline V72 & 13.361444 & 56.704306 & $15.658 \pm 0.009$ & $14.968 \pm 0.009$ \\
\hline V73 & 13.2766 & 56.581278 & $15.198 \pm 0.007$ & $14.346 \pm 0.006$ \\
\hline $\begin{array}{l}\text { V74 } \\
\text { V75 }\end{array}$ & $\begin{array}{l}13.318472 \\
1346361\end{array}$ & & $12.717 \pm 0.008$ & $12.369 \pm 0.007$ \\
\hline $\begin{array}{l}\text { V75 } \\
\text { V76 }\end{array}$ & $\begin{array}{l}13.346361 \\
13.236028\end{array}$ & $\begin{array}{l}56.683611 \\
56.526250\end{array}$ & $\begin{array}{l}16.608 \pm 0.023 \\
17.417 \pm 0.025\end{array}$ & $\begin{array}{l}15.530 \pm 0.010 \\
16.308+0.013\end{array}$ \\
\hline V77 & $\begin{array}{l}13.236028 \\
13.330972\end{array}$ & 56.665333 & $14.478 \pm 0.012$ & $\begin{array}{l}16.308 \pm 0.013 \\
13.684+0.009\end{array}$ \\
\hline V78 & 13.359389 & 56.709222 & $14.062 \pm 0.008$ & $12.353 \pm 0.011$ \\
\hline V79 & 13.269944 & 56.580111 & $17.225 \pm 0.040$ & $16.297 \pm 0.017$ \\
\hline V80 & 13.254417 & 56.56 & & \\
\hline V81 & 13.280000 & 56.604917 & $15.397 \pm 0.021$ & $14.473 \pm 0.007$ \\
\hline V82 & 13.304917 & 56.643000 & $15.239 \pm 0.007$ & $14.307 \pm 0.006$ \\
\hline V83 & 13.351472 & 56.712806 & $17.087 \pm 0.024$ & $16.205 \pm 0.013$ \\
\hline V84 & 13.302139 & 56.643861 & $12.534 \pm 0.005$ & $12.208 \pm 0.006$ \\
\hline V85 & 13.276806 & 56.608778 & $16.946 \pm 0.020$ & $15.738 \pm 0.011$ \\
\hline V86 & & 56.612972 & $15.255 \pm 0.008$ & $14.630 \pm 0.008$ \\
\hline V87 & 13.314944 & 56.671000 & $17.611 \pm 0.026$ & $16.157 \pm 0.019$ \\
\hline V88 & $\begin{array}{l}13.336028 \\
1336778\end{array}$ & $\begin{array}{l}56.702222 \\
56.559639\end{array}$ & $18.896 \pm 0.127$ & $17.407 \pm 0.031$ \\
\hline 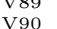 & $\begin{array}{l}13.236778 \\
13.323944\end{array}$ & $\begin{array}{l}56.559639 \\
56.687389\end{array}$ & $\begin{array}{l}13.344 \pm 0.007 \\
17.106+0.023\end{array}$ & $\begin{array}{l}12.876 \pm 0.007 \\
16.158+0.012\end{array}$ \\
\hline $\begin{array}{l}\text { V91 } \\
\text { V91 }\end{array}$ & $\begin{array}{l}13.323944 \\
13.255417\end{array}$ & 56.588111 & $15.329 \pm 0.009$ & $14.830 \pm 0.007$ \\
\hline V92 & 13.252111 & 56.584556 & & \\
\hline V93 & & & & \\
\hline V94 & 13.339917 & 56.7 & 10.0 & $9.662 \pm$ \\
\hline V95 & 13.295972 & 56.650639 & $13.924 \pm 0.006$ & $13.255 \pm 0.005$ \\
\hline V96 & 13.275417 & 56.621278 & $16.927 \pm 0.039$ & $15.382 \pm 0.009$ \\
\hline V97 & 13.2386 & 56.574 & $17.309 \pm 0.023$ & $16.365 \pm 0.017$ \\
\hline V98 & 13.280944 & 56.63 & 13.70 & $12.240 \pm 0.006$ \\
\hline V99 & 13.307 & 56.6812 & 16.26 & $15.258 \pm 0$. \\
\hline V100 & 13.218417 & 56.551361 & $11.400 \pm 0.006$ & $11.202 \pm 0.006$ \\
\hline $\begin{array}{l}\text { V101 } \\
\text { V102 }\end{array}$ & $\begin{array}{l}13.270972 \\
\end{array}$ & 56.628306 & $15.786 \pm 0.011$ & $14.820 \pm 0.009$ \\
\hline $\begin{array}{l}\text { V102 } \\
\text { V103 }\end{array}$ & $\begin{array}{l}13.230389 \\
13.268556\end{array}$ & $\begin{array}{l}56.574083 \\
56.630250\end{array}$ & $\begin{array}{ll}17.661 \pm 0.034 \\
17\end{array}$ & $16.249 \pm 0.013$ \\
\hline V104 & 13.269750 & 56.634861 & $15.717 \pm 0.009$ & $14.678 \pm 0.007$ \\
\hline V105 & 13.263083 & 56.630806 & $15.122 \pm 0.008$ & $14.588 \pm 0.007$ \\
\hline
\end{tabular}


Table 2. Continued.

\begin{tabular}{|c|}
\hline ID \\
\hline V112 \\
\hline $\mathrm{V} 113$ \\
\hline V114 \\
\hline V115 \\
\hline V116 \\
\hline V117 \\
\hline $\begin{array}{l}\text { V118 } \\
\text { V119 }\end{array}$ \\
\hline $\begin{array}{l}\text { V119 } \\
\text { V120 }\end{array}$ \\
\hline V121 \\
\hline V122 \\
\hline V123 \\
\hline V124 \\
\hline V125 \\
\hline $\begin{array}{l}\text { V126 } \\
\text { V127 }\end{array}$ \\
\hline $\begin{array}{l}\text { V128 } \\
\text { V }\end{array}$ \\
\hline V129 \\
\hline V130 \\
\hline $\begin{array}{l}\text { V131 } \\
\text { V132 }\end{array}$ \\
\hline $\begin{array}{l}\text { V132 } \\
\text { V133 }\end{array}$ \\
\hline $\begin{array}{l}\text { V133 } \\
\text { V134 }\end{array}$ \\
\hline V135 \\
\hline V136 \\
\hline V137 \\
\hline $\begin{array}{l}\text { V138 } \\
\text { V139 }\end{array}$ \\
\hline $\begin{array}{l}139 \\
\text { V140 }\end{array}$ \\
\hline V141 \\
\hline V142 \\
\hline V143 \\
\hline V144 \\
\hline $\begin{array}{l}\text { V145 } \\
\text { Y }\end{array}$ \\
\hline $\begin{array}{l}\text { V146 } \\
\text { V147 }\end{array}$ \\
\hline $\begin{array}{l}1478 \\
\text { V148 }\end{array}$ \\
\hline V149 \\
\hline V150 \\
\hline V151 \\
\hline V152 \\
\hline V153 \\
\hline V154 \\
\hline V155 \\
\hline V156 \\
\hline V157 \\
\hline $\begin{array}{l}\text { V158 } \\
\text { YV159 }\end{array}$ \\
\hline $\begin{array}{l}\text { V159 } \\
\text { V160 }\end{array}$ \\
\hline $\begin{array}{l}160 \\
\text { V161 }\end{array}$ \\
\hline V162 \\
\hline V163 \\
\hline V164 \\
\hline V165 \\
\hline $\begin{array}{l}\text { V166 } \\
\text { V167 }\end{array}$ \\
\hline $\begin{array}{l}\text { V167 } \\
\text { V168 }\end{array}$ \\
\hline V169 \\
\hline V170 \\
\hline V171 \\
\hline $\begin{array}{l}\text { V172 } \\
\end{array}$ \\
\hline $\begin{array}{l}\text { V173 } \\
\text { V174 }\end{array}$ \\
\hline $\begin{array}{l}\text { V1774 }\end{array}$ \\
\hline V176 \\
\hline V177 \\
\hline V178 \\
\hline V179 \\
\hline V180 \\
\hline V181 \\
\hline V182 \\
\hline V183 \\
\hline V184 \\
\hline V185 \\
\hline $\begin{array}{l}\text { V186 } \\
\text { V187 }\end{array}$ \\
\hline $\begin{array}{l}\text { V187 } \\
\text { V188 }\end{array}$ \\
\hline $\begin{array}{l}181 \\
\text { V189 }\end{array}$ \\
\hline V190 \\
\hline V191 \\
\hline V192 \\
\hline V193 \\
\hline V199 \\
\hline V195 \\
\hline V196 \\
\hline V197 \\
\hline V198 \\
\hline $\begin{array}{l}\text { V199 } \\
\text { P09 }\end{array}$ \\
\hline $\begin{array}{l}\text { V200 } \\
\text { V200 }\end{array}$ \\
\hline $\begin{array}{l}\text { V202 } \\
\end{array}$ \\
\hline V203 \\
\hline V204 \\
\hline V205 \\
\hline V206 \\
\hline $\begin{array}{l}\text { V207 } \\
\text { Y }\end{array}$ \\
\hline $\begin{array}{l}208 \\
\text { V209 }\end{array}$ \\
\hline V210 \\
\hline \\
\hline $\mathrm{V} 212$ \\
\hline $\mathrm{V} 213$ \\
\hline V214 \\
\hline V 215 \\
\hline V216 \\
\hline V217 \\
\hline V218 \\
\hline V219 \\
\hline
\end{tabular}



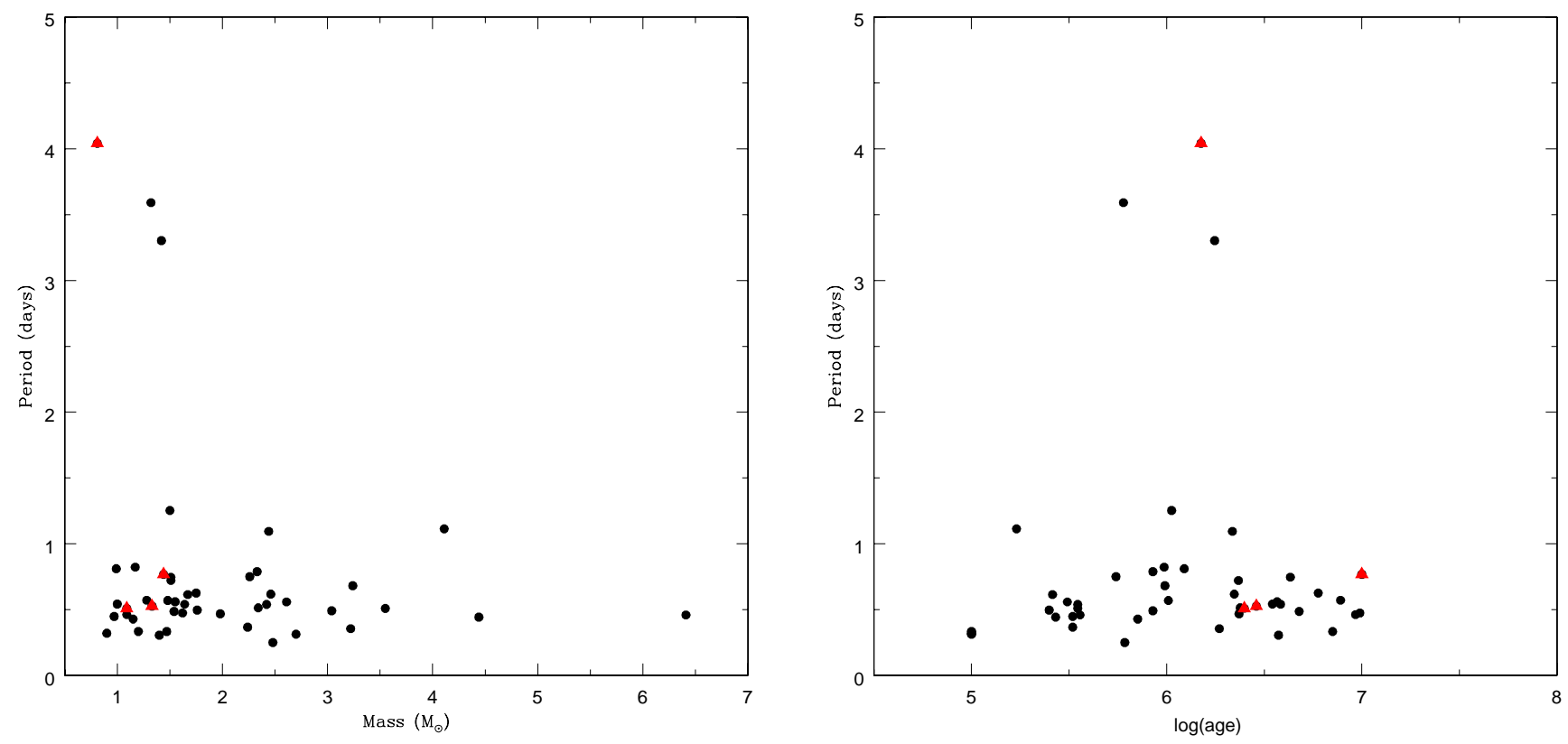

Figure 13. Rotation period of TT variables as a function of mass and age. Filled circles represent WTTSs while triangle shows CTTSs.

location of variable stars in the $H-R$ diagram has been estimated with the help of their intrinsic colour index $(B-V)_{0}$ and and $V$ magnitudes. The $U, B$, and $V$ magnitudes are available for $29 \mathrm{MS}$ periodic variables. The $U$ data of V145 is not available. The intrinsic colour $(B-V)_{0}$ of variable stars has been estimated using the Q-method (Gutiérrez-Moreno 1975). For determination of luminosity and temperature we require $V$ magnitudes, distance of the cluster and relations provided by Torres (2010). The details of the procedure for obtaining luminosity and temperature of the star can be found in Lata et al. (2019). We have plotted $\left(L / L_{\odot}\right)$ versus temperature diagram for $29 \mathrm{MS}$ variables in Fig. 12. Thus, based on location of variables in the $H-R$ diagram we have identified one variable as $\beta$ Cep candidate. The $\beta$ Cep stars are massive stars which are found to be located near the MS in the $H-R$ diagram. Their spectral types range from $\mathrm{O}$ to B. These variables pulsate with short periods. Multiple periods are often found in these stars (Jager et al. 1982). Star V167 is detected as $\beta$ Cep candidate with a period about 0.2 days in the present work. Five slowly pulsating B-type (SPB) candidates were also identified in the present work. SPB stars as their name suggests, are B spectral type stars. Their typical periods are in the range from 0.5 to 5 days and sometimes they show multiperiodicity. Waelkens (1991) studied SPB stars and named these early-type variables as 'SPB stars'. The characteristics (periods and shape of light curves) of present variables detected as SPB stars are consistent with the class. The present sample of MS variables also consists of 15 new class variables among MS variable members. The new class variable stars have properties similar to the ones discovered in NGC 3766 by Mowlavi et al. (2013). The periods and amplitudes of new class variable stars detected by Mowlavi et al. (2013) range from 0.1 to 0.7 days and 1 to $4 \mathrm{mmag}$, respectively. The present new class variable stars have periods between 0.25 to 0.80 days.
Additionally, we have also detected 4 stars which could be $\delta$ Scuti type variables. Their periods are found to be in the range of $\sim 0.22-0.25$ days. These $\delta$ Scuti stars are found to be pulsators which are located on/near the MS in the classical cepheid instability strip. These stars pulsate with period upto 5 or 6 hours. Three stars V94, V100 and V123 are neither lying on any instability regions of $H-R$ diagram nor in the location of new class variable. These stars might be field stars or belong to the PMS population.

In the present study we have detected 51 PMS stars. In general, the PMS objects are classified as T Tauri stars (TTSs) and Herbig Ae/Be stars. The TTSs are very young low mass stars (mass $\left.\lesssim 3 M_{\odot}\right)$ which are still contracting and moving towards the MS (Herbig 1957, 1977), whereas PMS stars of intermediate mass $\left(\gtrsim 3-10 M_{\odot}\right)$ with emission lines are considered as Herbig Ae/Be stars (Herbig 1960; Strom et al. 1972; Finkenzeller \& Mundt 1984). The TTSs are further classified as Weak line TTSs (WTTSs) and Classical TTSs (CTTSs) on the basis of the strength of the $H \alpha$ emission line (Strom et al. 1989) which is measured by its equivalent width $(\mathrm{EW})$. The WTTSs show weak $H \alpha(\mathrm{EW} \leqslant 10 \AA)$ emission with almost negligible infrared excess, whereas CTTSs generally have a strong $H \alpha$ emission line with $\mathrm{EW}>10 \AA$, large ultraviolet and infrared excesses. Both CTTSs and WTTSs show strong variability across all wavelengths and their brightness varies from a few minutes to years (see e.g., Appenzeller \& Mundt 1989). The brightness variation of TTSs are thought to occur most probably due to the presence of cool and/or hot spots on their surface (Herbst et al. 1987, 1994), which may originate due to several mechanisms such as circumstellar disk material, accretion and magnetic field (Herbst et al. 1994).

The cool spots on the surface of the stars are produced due to stellar magnetic fields on the photosphere and rotate with the star. If these spots are symmetrically distributed 

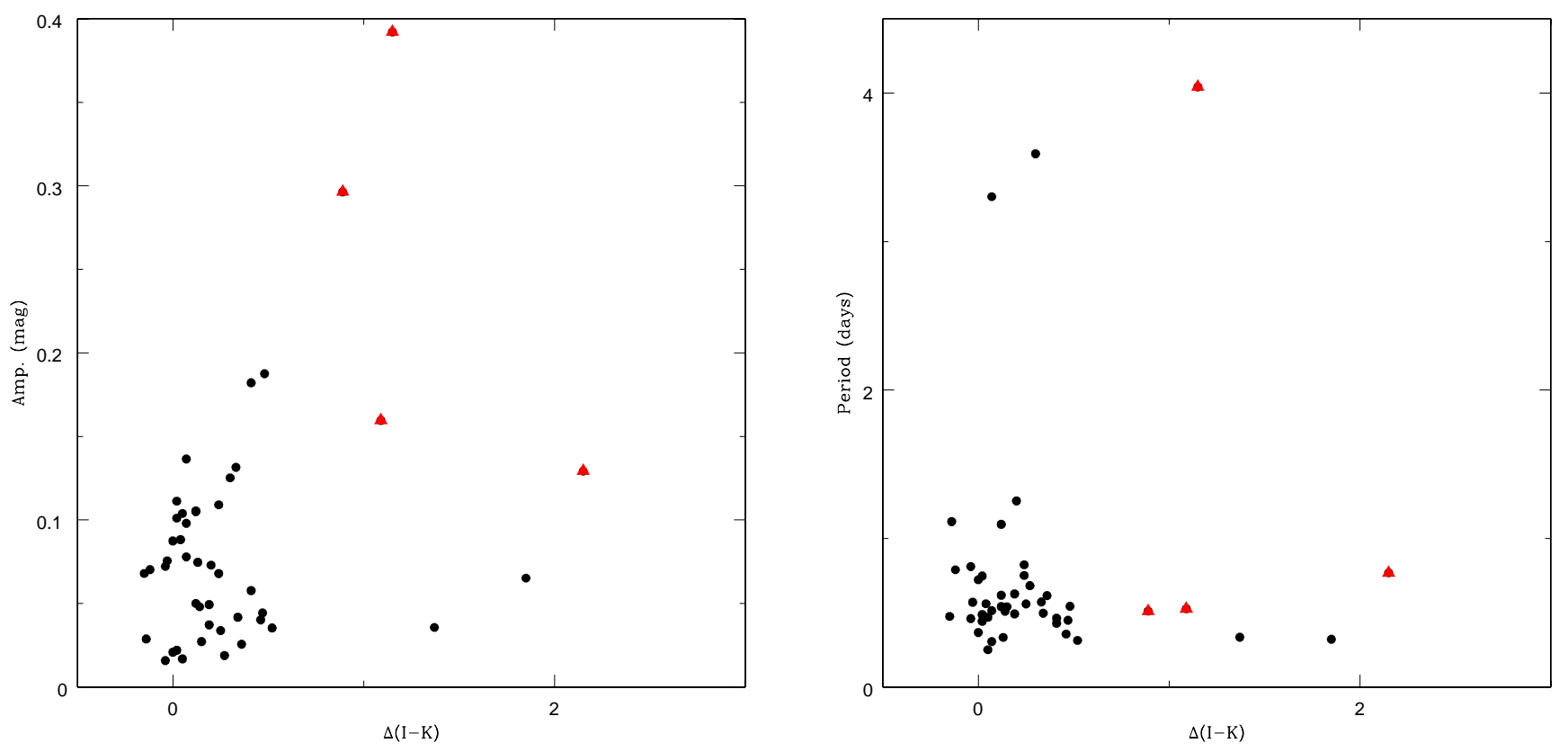

Figure 14. Amplitude and rotation period of TT variables versus $\Delta(I-K)$. The symbols are same as in Fig. 13 .

over the photosphere, periodic brightness variations are observed in the light curves of the stars. The cool spots on the photosphere of stars are responsible for brightness variation in WTTSs and these objects are found to be fast rotators as they have either thin or no circumstellar disk. WTTSs are characterized by smaller stellar flux variations (a few times $0.1 \mathrm{mag})$.

Accreting CTTSs, surrounded by circumstellar disks and have hot spots on the surface produced by accreting material from disks on to the stars, show a complex behaviour in their optical and NIR light curves (Scholz et al. 2009). Irregular or non-periodic variations are produced because of changes in the accretion rate. The time-scales of brightness variation range from hours to years. Although the hot spots cover a smaller area on the stellar surface, their higher temperature produces larger variability amplitude (Carpenter, Hillenbrand \& Skrutskie 2001). The Herbig Ae/Be stars also show variability as they cross the instability strip in the HR diagram on their way to the MS. However, exact cause of variability in Herbig Ae/Be is still not known.

Of 51 probable PMS stars identified in the present work, 42, 4 and 5 are classified as WTTSs, CTTSs and Herbig Ae/Be stars, respectively. The amplitudes of WTTSs ranges from $\sim 0.015 \mathrm{mag}$ to $\sim 0.19 \mathrm{mag}$, while periods of most WTTSs are found in the range of $\sim 0.25$ to $\sim 1.12$ days. The periods and amplitudes of CTTSs are found to vary $\sim 0.48$ to $\sim 4$ days and $\sim 0.13$ to $\sim 0.39 \mathrm{mag}$, respectively. The above results suggest that stars with disk i.e., CTTSs have relatively larger amplitudes than the WTTSs and indicate that variability in CTTSs could be due to the presence of hot spot on the stellar surface (see e.g., Carpenter, Hillenbrand \& Skrutskie 2001, Grankin et al. 2007, 2008, Lata et al. 2019). Similar results have been found in our earlier studies (Lata et al. 2019, 2016; Sinha et al. 2020).

Since the present sample of PMS stars is dominated by WTTSs (43 stars), we will further focus our analysis to understand the evolution of rotation period and amplitude of WTTSs identified in the present work. The masses and ages of PMS variables were estimated by comparing present observations with the theoretical models to study the evolution of amplitude and period of PMS stars. The procedure for estimation of mass and age is given in Lata et al. (2019) and Chauhan et al. (2009). For this, we have used PMS isochrones of Siess et al. (2000) in the age range of 0.1 to $10 \mathrm{Myr}$ with an interval of $0.1 \mathrm{Myr}$ and $V / V-I \mathrm{CMD}$ of the cluster. These theoretical isochrones corrected for the distance and reddening were compared with the locations of PMS stars in the $V / V-I$ CMD. Finally, we determine the mass and age of the PMS star corresponding to the closest isochrone on the CMD. The dependence of rotation period of TTSs (i.e., WTTSs and CTTSs) on age and mass is shown in Fig. 13 which reveals that rotation period of WTTSs does not depend either on age or mass of the stars. However, the slowest rotators in the present study are low mass $\left(\sim 1 M_{\odot}\right)$ stars.

WTTSs have either thin or no circumstellar disk, it will be useful to estimate the influence of circumstellar disks on period and amplitude of these variable stars. The studies available in the literature e.g., Herbst et al. (2000); Littlefair et al. (2005); Cieza \& Baliber (2007); Cody et al. (2018), have used various disk indicators, such as EW of the $\mathrm{H} \alpha$ emission line and Ca II triplet lines, $\Delta(H-K)$ and $\Delta(I-K)$ excess, disk fraction, etc. In the present study we have used $\Delta(I-K)$ excess which is defined as below (cf. Hillenbrand et al. 1998);

$$
\Delta(I-K)=(I-K)_{o b s}-\left(A_{I}-A_{K}\right)-(I-K)_{0}
$$

where $(I-K)_{o b s},(I-K)_{0}, A_{I}$ and $A_{K}$ are the observed colour, intrinsic colour of the star, interstellar extinction in the $I$ and $K$ bands, respectively. The $A_{V}$ value is taken as $0.99 \mathrm{mag}$ as determined using the relation $A_{V} / E(B-V)$. 
The values of $A_{I}$ and $A_{K}$ are estimated using the relations given by Cohen et al. (1981). To obtain intrinsic $(I-K)_{0}$ of variables we have used PMS evolutionary models of Siess et al. (2000) of a given mass and age. Fig. 14 displays the amplitude and period of TT variables as a function of $\Delta(I-K)$. It is evident from Fig. 14 that the majority of WTTSs have $\Delta(I-K)<0.3 \mathrm{mag}$, hence they can be considered as diskless sources. Apparently, no influence of $\Delta(I-K)$ either on amplitude or rotation of WTTSs is noticed. It is worthwhile to mention that a correlation between $\Delta(I-K)$ and amplitude as well as period of CTTSs in the sense that larger values of the disk indicator, i.e., $\Delta(I-K)$ corresponds to relatively larger amplitude variations has been reported in previous studies (e.g., Dutta et al. 2018, Sinha et al. 2019). Similarly, larger values of $\Delta(I-K)$ indicates longer rotation periods. An extensive study of ONC carried out by Herbst et al. $(2000,2002)$ reveals a strong correlation between rotation period and infrared excess, suggesting that the observed rotation period distribution could be due to the disk-locking mechanism. Similar results were reported by Edwards et al. (1993) and the physical interpretation proposed by the authors was that the disks slow the rotation of stars through magnetic interaction (Koenigl 1991; Ostriker \& Shu 1995). An increasing trend in disk fraction with period in ONC and NGC 2264 was reported by Cieza \& Baliber (2007). However, Littlefair et al. (2005) did not find any correlation between the $\mathrm{H} \alpha$ equivalent width or $(K-L)$ excess and the rotation period of the stars.

To check the dependence of $\Delta(I-K)$ on the stellar mass, we plot these parameters for all the PMS variables identified in the present study in Fig. 15 which reveals that higher values of NIR excess are associated with relatively low mass stars. This is consistent with the result that the slowest rotators in the present study are low mass $(\sim 1.0$ $\left.M_{\odot}\right)$ CTTSs (cf. Fig. 13).

Fig. 16 plots amplitude of variability as a function of mass of the PMS variables, which manifests that the amplitude decreases with the increase in mass.

Fig. 17 displays amplitude as a function of age. It is interesting to note that the amplitude of variability of WTTSs show an increase for relatively older stars. An inspection of figure 20 by Sinha et al. (2019) also indicates that the youngest WTTSs (age <1 Myr) in the Sh 2-170 H II region also show lower value of amplitudes as compared to older WTTSs. In the inset of Fig. 17 we plot data for WTTSs from our previous studies for the clusters Be 59, NGC 1893, NGC 7380 and Stock 8 (Lata et al. 2011, 2012, 2016 and 2019). The same trend has been noticed in previous data also. Grankin $(1999,2013)$ and Grankin et al. (2008) have shown that a small periodicity amplitude suggests a more uniform distribution of spots over the stellar surface, while a large amplitude is typical of the case where the spots are concentrated in one or two high-latitude regions, i.e., they are distributed highly non uniformly. These conclusions are also confirmed by the Doppler mapping of the surfaces of selected PMS stars (Grankin 2013). Thus, Fig. 17 seems to suggest that configuration/distribution of spots on the photosphere of WTTSs changes as they become older. The smaller amplitude for relatively larger mass $\left(\gtrsim 2.5 M_{\odot}\right)$ as noticed in Fig. 16 could be either due to dispersal of disk or uniform distribution of spots over stellar surface. It has been reported in our earlier studies that the disk dispersal

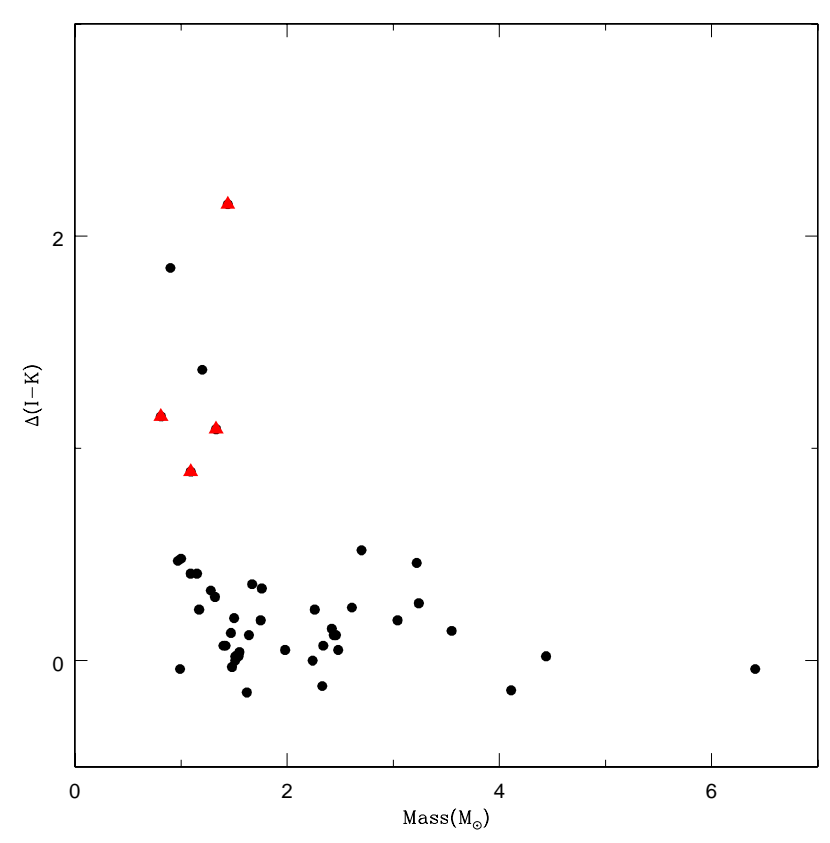

Figure 15. Excess $\Delta(I-K)$ as a function of mass for TT variables. The symbols are same as in Fig. 13.

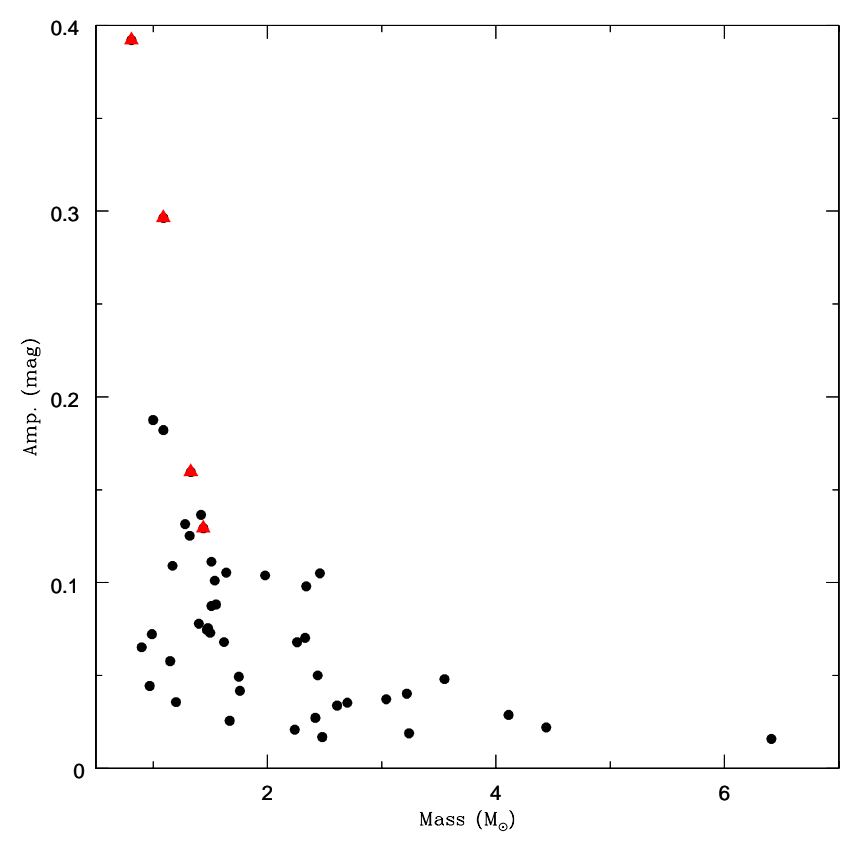

Figure 16. Amplitude of present $\mathrm{TT}$ variable candidates as a function of mass. The symbols are same as in Fig. 13.

mechanism is less efficient for relatively low mass stars (Lata et al. 2011, 2012, 2016).

The present study consists of 147 variables which could belong to the field population in the direction of NGC 281. Based on their light curves and variability characteristics, these stars could be RR Lyrae, $\delta$ Scuti, or binaries type vari- 


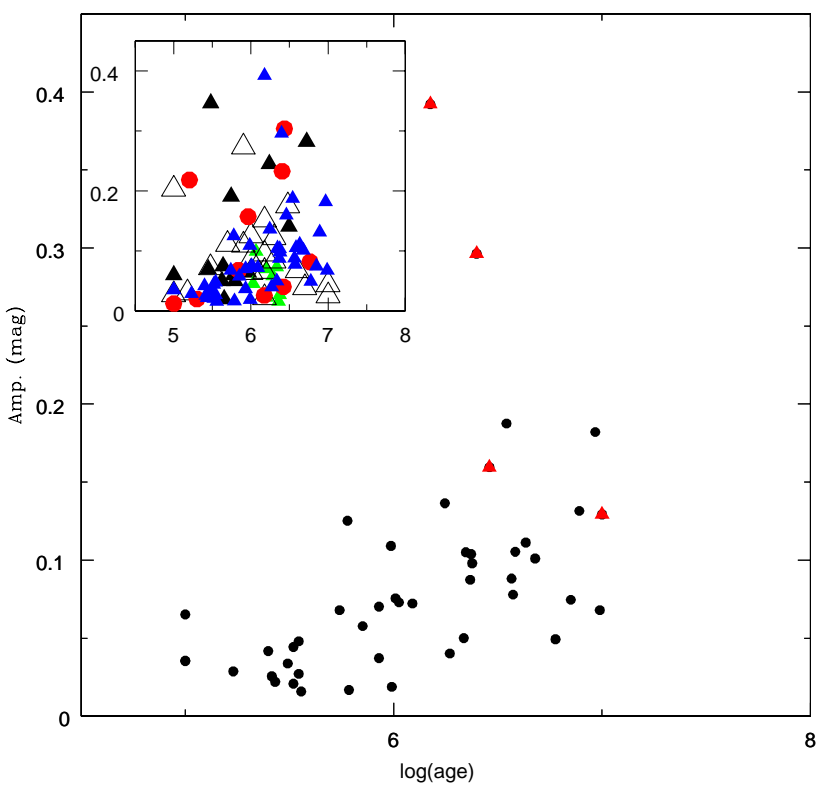

Figure 17. Amplitude of present TT variable candidates as a function of age. The symbols are same as in Fig. 13. Inset plots data for WTTSs taken from our earlier works (Lata et al. 2011, 2014, 2016 and 2019).

ables. As present study contains a few interesting variable stars which need to be characterized individually.

Star numbered V6 has a period of 0.157 days and an amplitude of $0.103 \mathrm{mag}$ which indicates that the star might be a $\delta$ Scuti variable. The shape of the light curve also resembles the light curve of $\delta$ Scuti variables having nearly sinusoidal nature.

Star V15 has $V$ magnitude about $18.81 \mathrm{mag}$ and it might belong to the field star population. With a period of 0.129 days and an amplitude of $0.261 \mathrm{mag}$, variable star V15 is suspected to be of the Ellipsoidal variables type which is a subgroup of the rotating variable stars. The light curve is W-shaped with minima portion substantially broader than the maxima (peak) which is quite sharp. The two clearly visible minimas might not be equally deep due to stronger limb-darkening effect on the pointed portion of the more elongated star.

Star V42 has a period of 0.735 days and an amplitude of 0.066 mag. It could possibly be RR Lyrae (RRab) type variable since it has a period which is similar to RRab type variables. In addition to this, it has asymmetric shaped light curve which is again a characteristic of RR Lyrae type variables.

Star V123 has a period of 0.76 days and amplitude of 0.049 mag. This star may be Cataclysmic variable of nova type as the light curve indicates that the amplitude decreases gradually as in case of cataclysmic variable.

Star V126 has a period of 0.06 days and 0.531 days with an amplitude of 0.015 mag. It is considered as PMS star (Herbig $\mathrm{Ae} / \mathrm{Be}$ ) in the present work. The shape of the light curve of this star indicates that this might be RS Canum Venaticorum eclipsing binary. In addition to this, amplitude of such variables are in the range of 0.01 to $0.6 \mathrm{mag}$ and the amplitude of this star also comes in this range.

In the present work star V152 is considered as a PMS star. Sharma et al. (2012) identified star V152 as a PMS source. However, this star in $J-H / H-K$ TCD does not lie in the region of class I, class II, II or Herbig Ae/Be. In fact, this is a bright star of $V$ magnitude around 11.3 mag, and in $V / V-I \mathrm{CMD}$ it is located on/near MS with other identified MS stars. It has a period of 0.46 days and amplitude of $0.016 \mathrm{mag}$, and its variability characteristics also reveals that it could be a slowly pulsating star.

The period of star V153 and V168 is 0.111 and 0.105 days, respectively and their amplitude is found as 0.018 mag. Based on variability characteristics, these stars could be of $\delta$ Scuti type variables.

\section{SUMMARY}

The present work detected 228 periodic variables in the field where young open cluster NGC 281 is located. The association of variables to the cluster has been discussed on the basis of TCDs, CMD and kinematic data. The membership probabilities of 223 stars have been determined using Gaia proper motion data. Using the present time series photometric data we have detected a number of PMS, MS and field variable stars. Eighty one of 228 variables are members, with 30 MS members and 51 PMS members. Based on their periods and light curves shape, and locations in the $H-R$ diagram we have characterized MS variable stars into different types of variability. These MS variables are classified as $\beta$ Cep, $\delta$ Scuti, SPB and new class type variables. The identified PMS variable stars are characterized as CTTSs, WTTSs and Herbig Ae/Be stars. The present study also indicates that the CTTSs vary with larger amplitude in comparison to the WTTSs. The masses and ages of PMS stars have been derived using CMD and PMS theoretical models. It is found that rotation period of WTTSs does not depend either on age or mass of the stars. Amplitude and mass of $\mathrm{TT}$ variables are found to be correlated in the sense that relatively massive stars $\left(\gtrsim 2.5 M_{\odot}\right)$ have smaller amplitudes. This could be due to lack of disk or uniform distribution of spots on the photosphere of the stars. We also note that the amplitude of variability of WTTSs increases with age, suggesting that the configuration/distribution of spots on the surface of WTTSs changes with their age. There are 147 variables which could belong to the field population. These field variables could be RR Lyrae, $\delta$ Scuti, or binaries type variables.

\section{ACKNOWLEDGMENT}

We are thankful to the referee for careful reading of the paper and valuable suggestions/comments.

\section{AVAILABILITY OF DATA}

The data underlying this article will be shared on reasonable request to the corresponding author.

The data underlying this article are available in 2MASS 
(https://vizier.u-strasbg.fr/viz-bin/VizieR?-source=II/246) and Gaia (https://gea.esac.esa.int/archive/).

\section{REFERENCES}

[] Appenzeller I., \& Mundt R., 1989, Astron. Astrophys. Rev. 1, 291

[] Arcos C., Kanaan S., Chavez J., Vanzi L., Araya I., Cure M., 2018, MNRAS, 474, 5287

[] Balona L. A., Pigulski A., Cat P. De, Handler G., Gutiérrez-Soto J., Engelbrecht C. A., Frescura F., Briquet M., et al., 2011, MNRAS, 413, 2403

[] Bessell M. S., Brett J. M., 1988, PASP, 100, 1134

[] Burger M., de Jager C., van den Oord, G. H. J., Sato N., 1982, A\&A, 107, 320

[] Carpenter J. M., Hillenbrand L. A., Skrutskie M. F., 2001 AJ, 121, 3160

[] Cabrera-Cano J. and Alfaro E. J., 1990, A\&A, 235, 94

[] Chauhan N., Pandey A. K., Ogura K. et al, 2009, MNRAS, 396, 964

[] Cutri R. M. et al., 2003 , 2MASS All Sky Catalog of Point Sources, VizieR Online Data Cata$\log$, University of Massachusetts and Infrared Processing and Analysis Center (IPAC/California Institute of Technology), 2246, 0 https://vizier.u-strasbg.fr/vizbin/VizieR?-source=II $/ 246$

[] Cohen J. G., Persson S. E., Elias J. H., Frogel J. A., 1981, ApJ, 249, 481

[] Cody A. M., Hillenbrand L. A. 2018, AJ, 156, 71

[] Cieza Lucas, Baliber Nairn, 2007, ApJ, 671, 605

[] Dutta S., Mondal S., Joshi S., Das R., 2019, BSRSL, 88,103

[] de Jager C., Sato, N., Burger M., Neven L., 1982, Ap\&SS, 83, 411

[] Edwards S., Strom S. E., Hartigan P., Strom K. M., Hillenbrand L. A., Herbst W., Attridge J., Merrill K. M., Probst R., Gatley I., 1993, AJ, 106, 372

[] Elmegreen B. G. \& Lada C. J., 1978, ApJ, 219, 467

[] Finkenzeller U., Mundt R., 1984, A\&AS, 55, 109

[] Gaia Collaboration et al. 2018, A\&A, 616, 13 https://gea.esac.esa.int/archive/

[] Getman K. V., Feigelson E. D., Sicilia-Aguilar A., et al., 2012, MNRAS, 426, 2917

[] Girardi L., Bertelli G., Bressan A., Chiosi C., Groenewegen M. A. T., Marigo P., Salasnich B., Weiss A., 2002, A\&A, 391, 195

[] Grankin K. N., Melnikov S. Yu., Bouvier J., Herbst W., Shevchenko V. S., 2007, A\&A, 461, 183

[] Grankin K. N., Bouvier J., Herbst W., Melnikov S. Y., 2008, A\&A, 479, 827

[] Grankin K. N., 2013, Astronomy Letters, 2013, Vol. 39, 446

[] Grankin K. N., 1999, Astron. Lett. 25, 526

[] Gutiérrez-Moreno A., 1975, PASP 87, 805

[] Herbig G. H., 1960, ApJS, 4, 337

[] Herbst W., Herbst D. K., Grossman E. J., Weinstein D., 1994, AJ, 108, 1906

[] Herbst W., Booth J. F., Korett F. L., et al, 1987, AJ, 94, 13

[] Herbst W., Bailer-Jones C. A. L., Mundt R., Meisenheimer K., Wackermann R., 2002, A\&A, 396, 513
[] Herbst W., Maley J. A., Williams E. C., 2000, AJ, 120, 349

[] Ivers Carol B., Booker M., Piper M., Powers L., Ali B., Wolk S. J., 2014, NITARP, AAS, 22324419

[] Jose J., Pandey A. K., Samal M. R., Ojha D. K., Ogura K., Kim J. S., Kobayashi N., Goyal A., Chauhan N., Eswaraiah C., 2013, MNRAS, 432, 3445

[] Koenigl Arieh, 1991, ApJ, 370L, 39

[] Landolt A. U., 1992, AJ, 104, 340

[] Lata S., Pandey A. K., Maheswar G., Mondal S. and Kumar B., 2011, MNRAS, 418, 1346

[] Lata S., Pandey A. K., Chen W. P., Maheswar G. and Chauhan N., 2012, MNRAS, 427, 1449

[] Lata S., Pandey A. K., Panwar N. et al., 2016, MNRAS, 456,2505

[] Lata Sneh, Pandey Anil K., Kesh Yadav Ram, et al. 2019, AJ, 158, 68

[] Lomb N. R., 1976, Ap\&SS, 39, 447

[] Littlefair S. P., Dhillon V. S., Martín E. L., 2005, A\&A, 437, 637

[] Meyer M. R., Calvet N., Hillenbrand L. A., 1997, AJ, 114,288

[] Mowlavi N., Barblan F., Saesen S., Eyer L., 2013, A\&A, 554,108

[] Ostriker Eve C., Shu Frank H., 1995, ApJ, 447, 813

[] Sato M. et al., 2008, PASJ, 60, 975

[] Scholz A., Eislőffel J., Mundt R., 2009, MNRAS, 400, 1548

[] Scargle J. D., 1982, ApJ, 263, 835

[] Sesar B. et al, 2007, AJ, 134, 2236

[] Sharma Saurabh, Pandey Anil K., Pandey Jeewan C., Chauhan Neelam, Ogura Katsuo, Ojha Devandra K., Borrissova Jura, Mito Hiroyuki, Verdugo Thomas, Bhatt Bhuwan C., 2012, PASJ, 64, 107

[] Siess L., Dufour E., Forestini M., 2000, A\&A, 358, 593

[] Sinha Tirthendu, Sharma Saurabh, Pandey A. K., Yadav R. K., Ogura K., Matsunaga N., Kobayashi N., Bisht P. S., Pandey R., Ghosh A., 2020, MNRAS, 493, $267 \mathrm{~S}$

[] Stetson P. B., 1987, PASP, 99, 191

[] Stetson P. B., 1992, J. R. Astron. Soc. Can., 86, 71

[] Strom S. E., Strom K. M., Yost J., et al, 1972, ApJ, 173, L65

[] Strom K. M., Strom S. E., Edwards S., Cabrit S., Skrutskie, M. F. 1989, AJ, 97, 1451

[] Torres G., 2010, AJ, 140, 1158

[] Waelkens C., 1991, A\&A, 246, 453 